\title{
AN OVERVIEW OF RESULTS FROM RPI ON IMAGE
}

\author{
J. L. GREEN ${ }^{1}$ and B. W. REINISCH ${ }^{2}$ \\ ${ }^{1}$ NASA Goddard Space Flight Center, Greenbelt, MD \\ ${ }^{2}$ Center for Atmospheric Research, University of Massachusetts, Lowell, MA
}

\begin{abstract}
The Radio Plasma Imager (RPI) on the Imager for Magnetopause-to-Aurora Global Exploration (IMAGE) spacecraft was designed as a long-range magnetospheric radio sounder, relaxation sounder, and a passive plasma wave instrument. The RPI is a highly flexible instrument that can be programmed to perform these types of measurements at times when IMAGE is located in key regions of the magnetosphere. RPI is the first radio sounder ever flown to large radial distances into the magnetosphere.

The long-range sounder echoes from RPI allow remote sensing of a variety of plasmas structures and boundaries in the magnetosphere. A profile inversion technique for RPI echo traces has been developed and provides a method for determining the density distribution of the plasma from either direct or field-aligned echoes. This technique has enabled the determination of the evolving density structure of the polar cap and the plasmasphere under a variety of geomagnetic conditions. New results from RPI show that the plasmasphere refills in slightly greater than a day at $\mathrm{L}$ values of 2.8 and that ion heating is probably playing a major role in the overall density distribution along the field-line. In addition, RPI's plasma resonance observations at large radial distances over the polar cap provided in situ measurements of the plasma density with an accuracy of a few percent. For the first time in the magnetosphere, RPI has also observed the plasma D resonances.

RPI's long antennas and its very low noise receivers provide excellent observations in the passive receive-only mode when the instrument measures the thermal plasma noise as well as natural emissions such as the continuum radiation and auroral kilometric radiation (AKR). Recent passive measurements from RPI have been compared extensively with images from the Extreme Ultraviolet (EUV) imager on IMAGE resulting in a number of new discoveries. For instance, these combined observations show that kilometric continuum can be generated at the plasmapause from sources in or very near the magnetic equator, within a bite-out region of the plasmasphere. The process by which plasmaspheric bite-out structures are produced is not completely understood at this time.

Finally, RPI has been used to successfully test the feasibility of magnetospheric tomography. During perigee passages of the Wind spacecraft, RPI radio transmissions at one and two frequencies have been observed by the Waves instrument. The received electric field vector was observed to rotate with time due to the changing density of plasma, and thus Faraday rotation was measured. Many future multi-spacecraft missions propose to use Faraday rotation to obtain global density pictures of the magnetosphere.
\end{abstract}

\section{Introduction}

The IMAGE spacecraft was launched on March 25, 2000 into a highly elliptical polar orbit with initial geocentric apogee of 8.22 Earth radii $\left(\mathrm{R}_{\mathrm{E}}\right)$ and perigee altitude of $1000 \mathrm{~km}$. The Radio Plasma Imager (RPI) instrument on IMAGE is a highly flexible radio sounder that transmits and receives coded radio frequency 


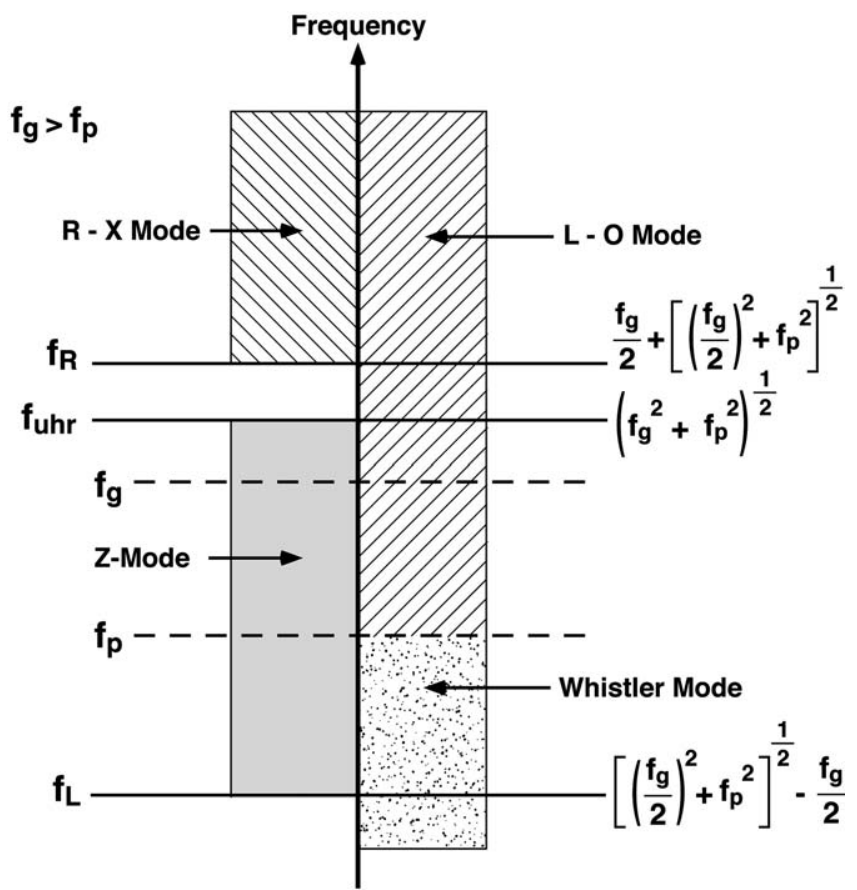

Figure 1. Example of the propagation modes of RPI echoes during a complete sweep in sounder frequency for a plasma in which $f_{g}>f_{p}$. The cutoff or resonance equations for a cold plasma are also shown. Waves can propagate in frequency ranges where there is a common shading.

pulses in the frequency range from $3 \mathrm{kHz}$ to $3 \mathrm{MHz}$. RPI utilizes three orthogonal dipole antennas of $325 \mathrm{~m}$ ( $\mathrm{X}$ axis), $500 \mathrm{~m}$ ( $\mathrm{Y}$ axis), and $20 \mathrm{~m}$ ( $\mathrm{Z}$ axis). The $\mathrm{X}$ axis antenna was originally extended to $500 \mathrm{~m}$ but was shortened to $325 \mathrm{~m}$ when it apparently collided with a meteor or debris on October 3, 2000. The X axis dipole is used for transmission while all antennas are used for the reception of the return echoes and for making passive radio measurements. For more details on the RPI instrument, see Reinisch et al. [2000].

The RPI is programmed to perform radio sounding over a specified range of frequencies during passage near and through key magnetospheric regions such as the plasmasphere, the high and low altitude polar cap, and the polar cusp. The measurement programs are designed to provide sounding data that start at frequencies well below the local electron plasma frequency $\left(f_{p}\right)$ and electron gyrofrequency $\left(f_{g}\right)$ and extend to frequencies well above the local upper hybrid frequency $\left(f_{\text {uhr }}\right)$. Figure 1 shows the fundamental plasma modes of the echoes when IMAGE is in a region where $f_{g}>f_{p}$ (i.e. polar cap). As the sounding frequency increases the resulting echoes propagate in the whistler, $\mathrm{Z}$, and the L-O and R-X modes. The frequency range of each mode has been shaded to more easily distinguish between them. These modes will have lower and, sometimes, upper frequency limits to their propagation that are called cutoffs or resonances as described by Stix [1962]. 


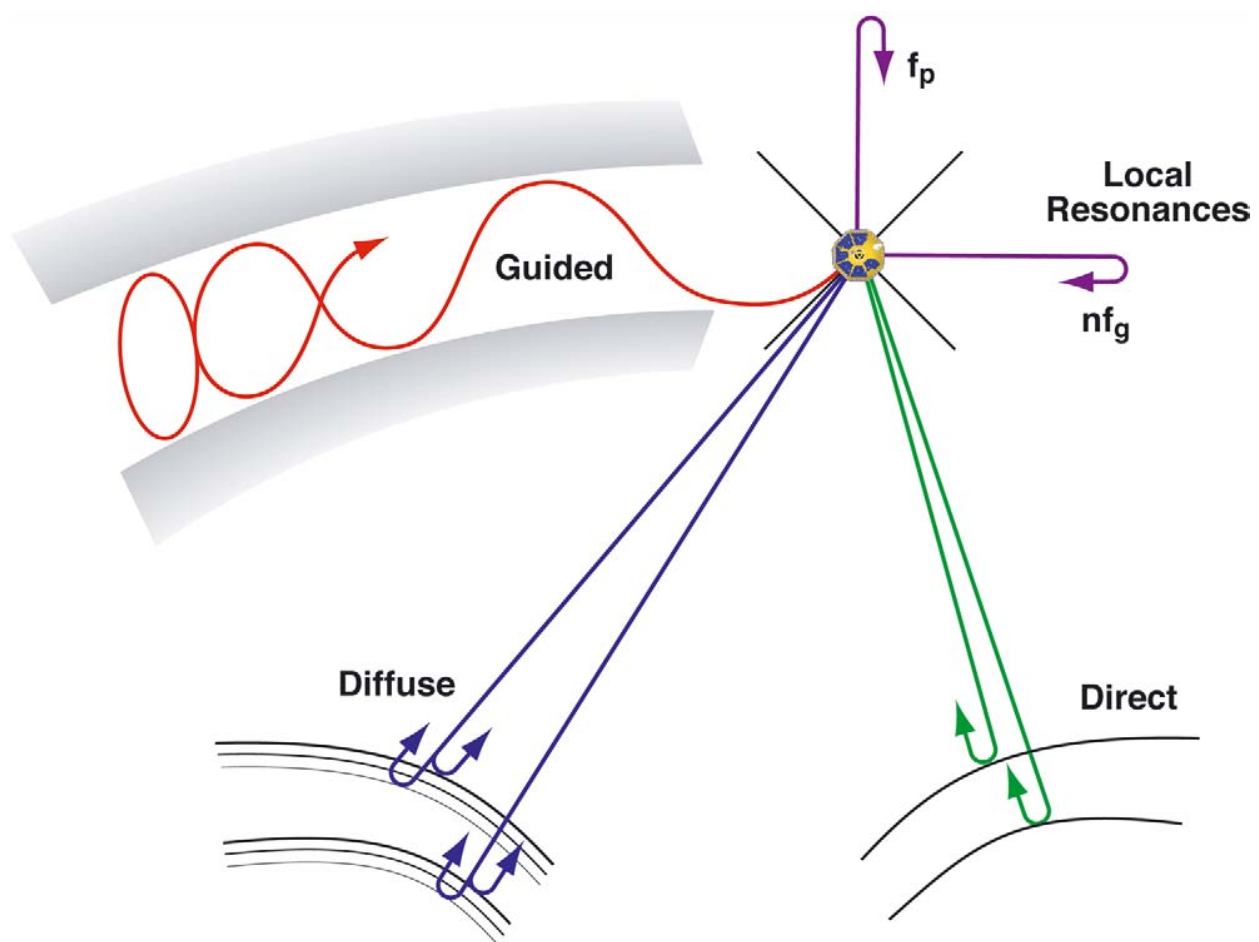

Figure 2. A schematic of the types of echoes observed by RPI showing the long range guided, diffuse, and direct echoes along with the sort range local resonances at the $f_{p}$ frequency and at the harmonics (n) of the $f_{g}$ frequency.

Once the propagation mode of an RPI echo has been identified, the knowledge of its propagation cut off frequency provides us with a powerful analysis tool to remotely probe plasma structures and, from a sequence of such observations, magnetospheric dynamics.

\section{Radio Sounding Measurements}

RPI observes several different types of magnetospheric echoes. These are shown in Figure 2. Long-range echoes have been classified as either direct, diffuse, or guided echoes depending on their propagation paths. For R-X or L-O mode waves, direct echoes occur from reflections in a plasma when the index of refraction (which is a function of frequency) goes to zero. For RPI to receive an echo, the direction of propagation (group velocity) of the reflecting wave is also parallel to the gradient of the index of refraction at the reflection point. Diffuse echoes are the result of plasma irregularities in the reflection region. This causes some of the radiation to be reflected at different distances and at a variety of angles. Guided echoes have also been observed. The conditions in the magnetoplasma are such 
A January 19, 2001

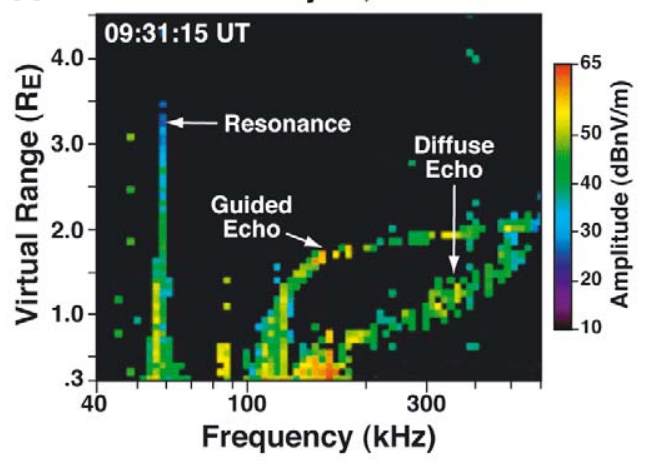

B

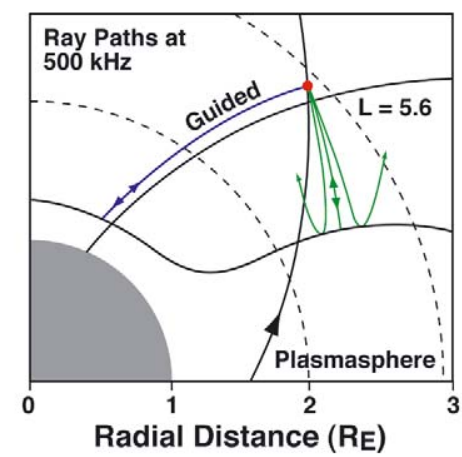

C

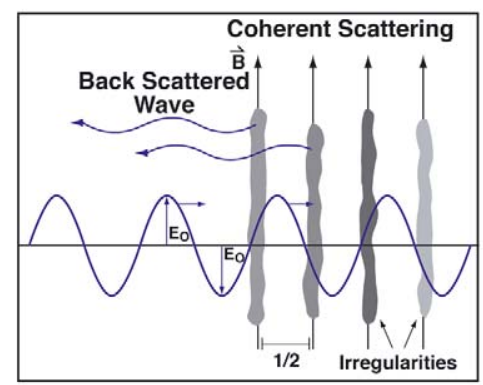

Figure 3. Panel A shows a typical RPI plasmagram with each of the main echo features labeled. Panel B illustrates the directions of the observed guided and direct echoes. As shown in panel A, the direct echoes from the plasmapause are spread in range or diffuse and are therefore reflecting from a series of irregularities as shown in panel C. This figure was adapted from Carpenter et al., 2002. 
that these waves are guided along the magnetic field reflecting at the distant cutoff and returning virtually along the same ray trajectory. These echoes have also been referred to as field-aligned or ducted. Frequently RPI observes short-range echoes that travel on paths in which the local plasma interacts quickly with the radiation and returns the echoes to the spacecraft typically within a very short time period. Since these echoes occur at resonance frequencies of the local plasma they are commonly referred to as local resonances.

The primary presentation of RPI echo measurements are in the form of plasmagrams, which are the magnetospheric analog of ionograms. A plasmagram is a plot of echo amplitude as a function of frequency and virtual range (derived from an echo's delay time). Panel A of Figure 3 is a plasmagram taken when the IMAGE spacecraft was just outside of the plasmasphere [from Carpenter et al., 2002]. The virtual range corresponds to $\mathrm{ct} / 2$ where $\mathrm{c}$ is the speed of light and $\mathrm{t}$ is the echo delay time. The echo intensity is color-coded. The three basic types of RPI echoes (diffuse, guided, and resonance) are observed in panel A. Direct and guided echoes typically have very distinct characteristics when presented in plasmagram form. RPI direct echoes typically show range or frequency spreading while field-aligned echoes always have discrete traces in plasmagrams and in many cases multiple traces over the same frequency range. In addition, direction-finding measurements show the field-aligned nature of the guided echoes. Based on direction measurements and the virtual range determination the ray paths of the diffuse and guided echoes are shown schematically in panel B of Figure 3. As shown in panel A, the diffuse echoes are spread in virtual range at many frequencies. These echoes are from the plasmapause. As shown in panel $\mathrm{C}$ of Figure 3 the range spreading can be attributed in part to coherent scattering from cross-field electron density irregularities of $<10 \%$ of the background density that range in size from $200 \mathrm{~m}$ to greater than $10 \mathrm{~km}$ [Carpenter et al., 2002]. The frequent occurrence of diffuse echoes from the plasmapause suggests that the field-aligned irregularities probably exist even during relatively quiet times. These field-aligned irregularities may be due to a variety of processes resulting from variations in local wave phenomena and/or cross-field fluctuations in ionospheric outflow during times of plasmaspheric refilling. The guided echoes in panel A are due to nearly field-aligned propagation and are coming from reflections in the closest hemisphere and will be discussed in more detail in the next section. Finally, a local gyroresonance is also seen in the plasmagram of panel A.

\subsection{Plasmaspheric Guided Echoes}

One of the most exciting results from RPI is the large number of guided X-mode echoes that are observed within many regions of the magnetosphere [Reinisch et al., 2001a, 2001b; Fung et al., 2003]. Panel B of Figure 4 shows a plasmagram with multiple guided echoes and one diffuse echo trace during a time when IMAGE was within the plasmasphere. The schematic in Panel A of Figure 4 shows the cor- 

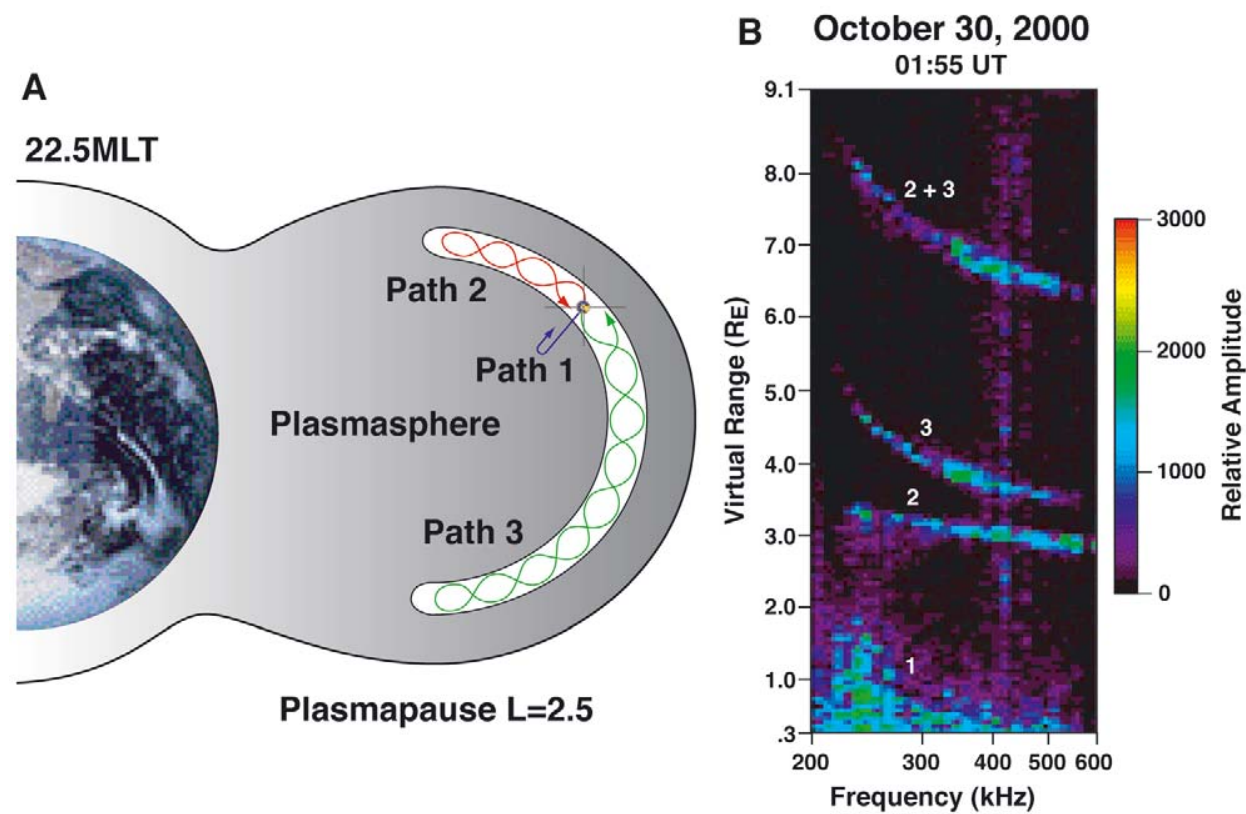

Figure 4. The plasmagram shows diffuse echoes from the plasmasphere (path 1 echoes) and a series of field-aligned guided echoes (paths 2 and 3). Panel A is a schematic of the propagation paths of the guided echoes. The echoes at the greatest values of the virtual range have traveled both path 2 and 3 before being measured by RPI. Panel B shows the plasmagram.

responding echo propagation paths. Path 1 echoes are diffuse with significant range spreading and are reflections from irregular density structures below the spacecraft. Paths 2 and 3 are guided X-mode echoes following the geomagnetic field to both the northern and southern hemispheres with reflection at their local cutoff frequencies (see the equation for $f_{R}$ in Figure 1). The long-range traces labeled $2+3$ in the plasmagram of Figure 4 are formed by echoes that propagated along path 2 then propagated along path 3 (and along path 3 then along path 2 ) before being measured by RPI. This can easily be seen by looking at the virtual ranges of the guided echoes at any frequency in Figure 4 panel B. For example, at $350 \mathrm{kHz}$ the path 2 echo was measured at a delay time corresponding to a propagation distance (in virtual range) of $3 \mathrm{R}_{\mathrm{E}}$, and for path 3 about $4 \mathrm{R}_{\mathrm{E}}$. The echo labeled $2+3$ combines these two virtual ranges for a propagation path of about $7 \mathrm{R}_{\mathrm{E}}$ in virtual range.

The technique used for determining the electron densities from RPI echoes is a variation on that developed by Huang and Reinisch [1982] for the ionosphere and is discussed in greater detail in Reinisch et al. (2001a). In brief, using the measured virtual ranges for a set of $\mathrm{X}$-mode echoes over a frequency range and assuming a model magnetic field the inversion of the index of refraction determines, through a least squares fitting process, a density profile along the ray path or in the case of the guided echoes, along the geomagnetic field. 


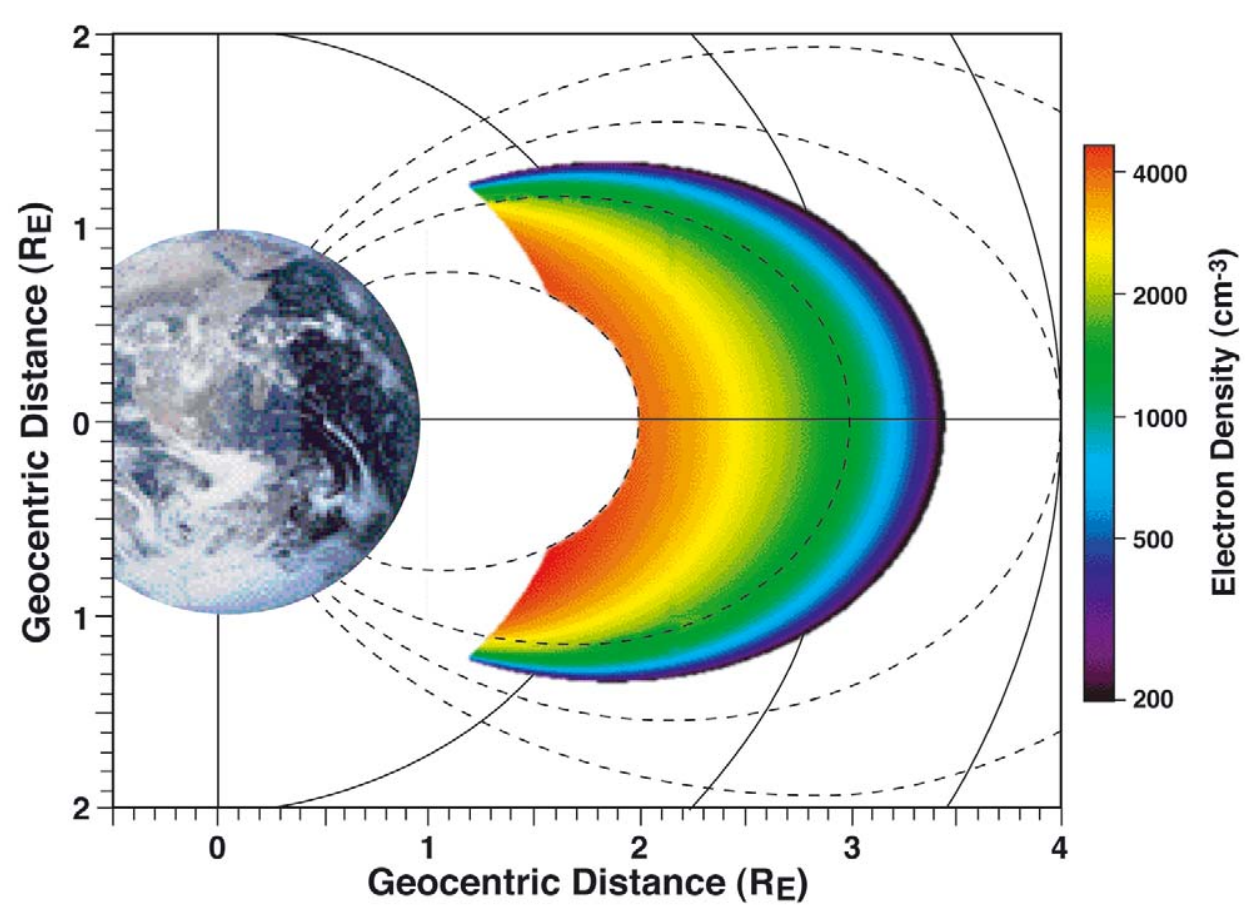

Figure 5. A quiet day empirical plasmasphere model has been derived from the inversion of RPI guided echoes on March 27, 2001 to obtain this 2-dimensional field-aligned density structure of the plasmasphere.

The electron density $\left(\mathrm{N}_{\mathrm{e}}\right)$ along a geomagnetic field line can be determined from the observed guided echoes within the plasmasphere region for successive RPI plasmagrams. A set of such profiles provides an empirical $\mathrm{N}_{\mathrm{e}}$ model of the plasmasphere as illustrated in Figure 5 [Reinisch et al., 2002]. Profiles from plasmagrams during low $\mathrm{K}_{\mathrm{p}}$ periods between March 27 and 30, 2001 when the plasmaspheric flux tubes were filled are used to create the meridian plane density distribution shown in Figure 5. Using this quiet day empirical model as a standard for comparison, the time it takes for the plasmasphere to refill after the March 31, 2001 storm period can then be determined.

The March 31, 2001 storm was so strong that the enhanced cross tail electric field reduced the $\mathrm{L}$ value of the plasmapause to about 2.3 thereby emptying plasmaspheric flux tubes all the way out to the pre-storm plasmapause at $\mathrm{L}=5$. The RPI observations of guided echoes after this very large geomagnetic storm are shown in panel A of Figure 6 (as an example of one out of a succession of plasmagrams). Inverting the guided echoes observed at an $\mathrm{L}$ value of 2.7 gives a density profile shown in panel B of Figure 6 . The density along the same L value from the quiet day empirical model of Figure 5 is also shown. A large difference in $\mathrm{N}_{\mathrm{e}}$ is found in this comparison indicating the extensive loss of plasma that occurred during the geomagnetic storm period. Since plasmasphere filling is a process that 


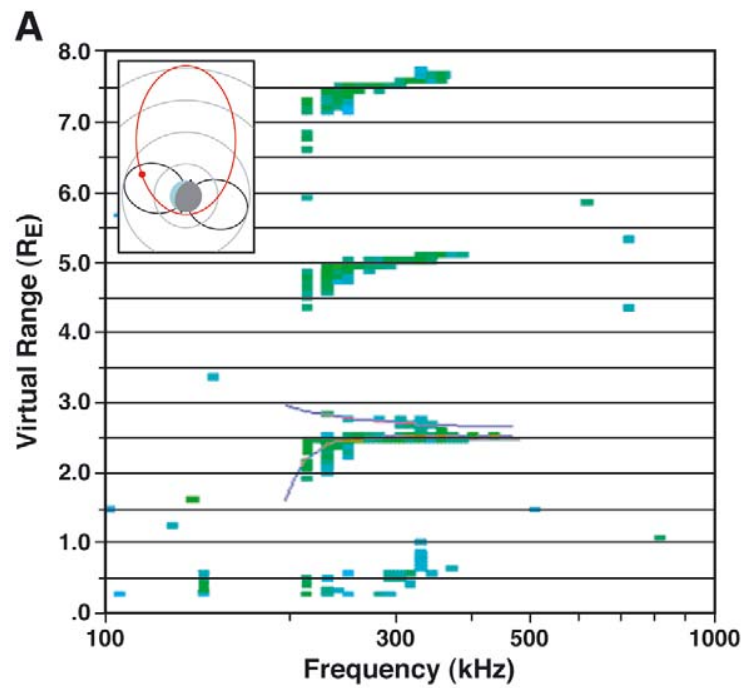

B

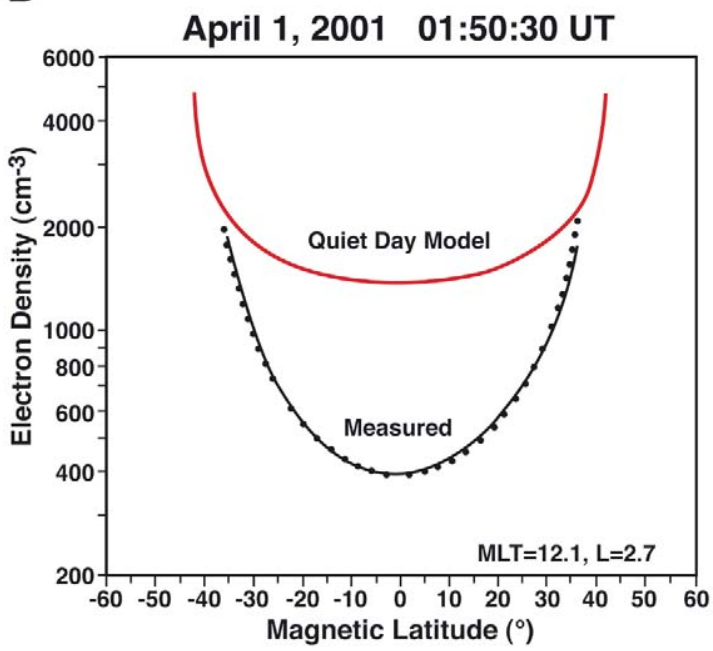

Figure 6. Panel A is a plasmagram on April 1, 2001 right after the very large magnetic storm of March 31, 2001 showing a series of guided echoes. Panel B is the density as a function of the magnetic latitude of both the quiet day model and the measured density resulting from the inversion of the guided echoes from Panel A showing the density structure of the plasmasphere at an $\mathrm{L}=2.7$. The plasmasphere is in a state of refilling.

can take a day or more, successive IMAGE passes through the plasmasphere can be used to monitor the filling process. The IMAGE/EUV observations clearly shows that the plasmasphere was stripped of cold plasma down to very low $\mathrm{L}$ values at all local times during this geomagnetic storm. A plasma convection tail was also observed by EUV in the late evening local time sector; however, the RPI observations were made along the orbit plane, which was primarily in the noon- 


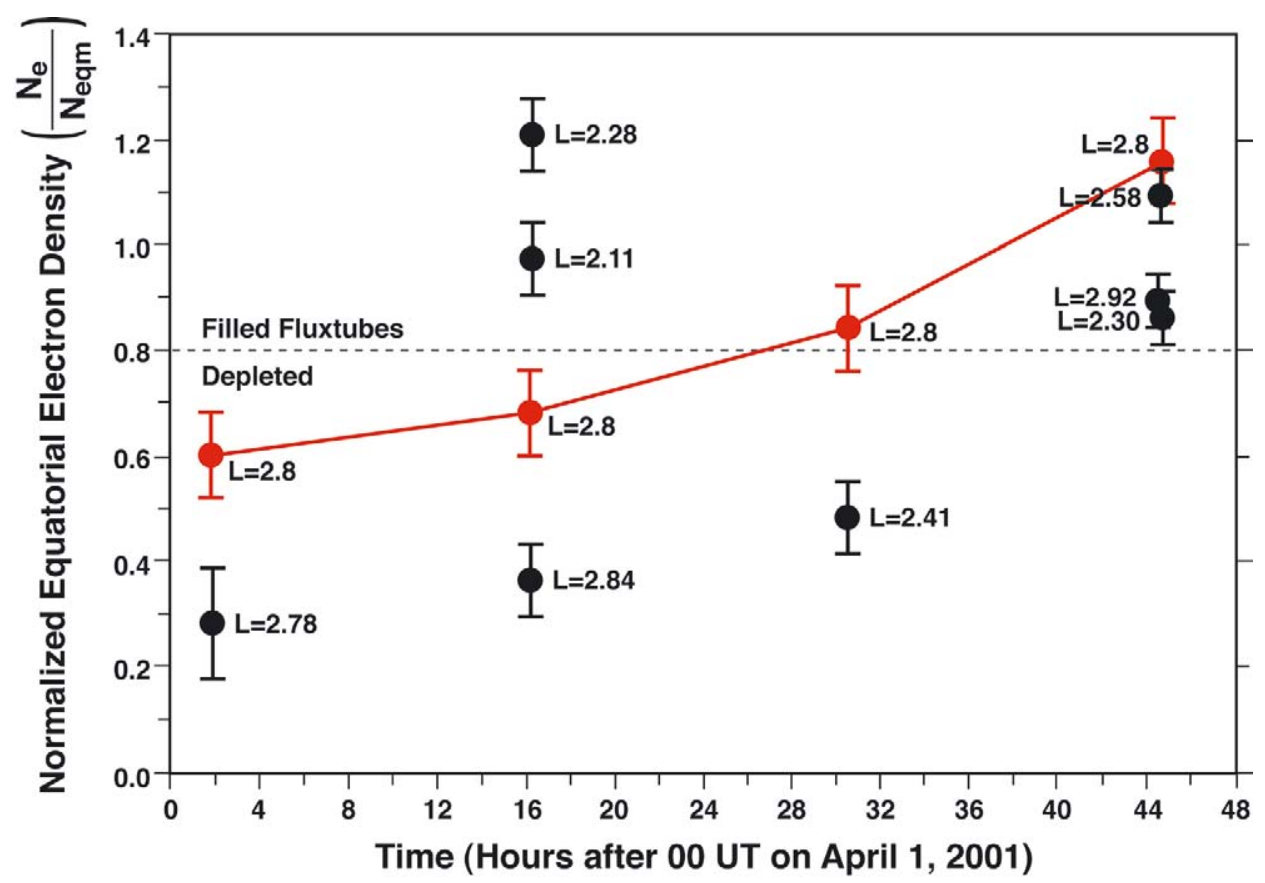

Figure 7. A plot of the $\mathrm{N}_{\mathrm{e}}$ at the equator normalized to the quiet day model as measured by RPI from four passes through the plasmasphere at a number of $\mathrm{L}$ shells. The red line shows the measured results from an $\mathrm{L}=2.8$ and illustrates that this flux tube took approximately 28 hours to fill.

midnight meridian avoiding any region of enhanced plasmaspheric material that would adversely effect the determination of the filling rate.

Figure 7 shows the RPI derived equatorial $\mathrm{N}_{\mathrm{e}}$ normalized by the quiet day equatorial $\mathrm{N}_{\mathrm{e}}$ from four consecutive passes of IMAGE through the plasmasphere. Since the IMAGE orbital period is approximately 14 hours, 'snapshots' of plasmaspheric filling can be obtained. The inner plasmasphere, below about $\mathrm{L}$ of 2.3 , shows no depletion from the storm while the equatorial densities at higher $\mathrm{L}$ values undergo extensive depletion. The refilling process at $\mathrm{L}=2.8$ started at approximately 1600 UT on April 1 and, as shown in Figure 7, is complete by about 2000 UT on April 2. The refilling of plasma in the plasmasphere at an $\mathrm{L}$ value of 2.8 is therefore completed in less than about 28 hours. These observations are consistent with those of Park [1974], who determined refilling times from whistler observations.

The RPI observations of guided echoes also provide a unique capability to compare RPI derived field-line density distributions with plasmasphere transport models. Recently, comparisons by Tu et al. (2003) of RPI derived field-line density distributions with the field-line inter-hemispheric plasma (FLIP) model shows good agreement only for the equatorial region and that higher densities are found at higher latitudes. The agreement can be improved by invoking direct heating of ions in the plasmasphere at the equator during the time of plasmasphere refilling. 

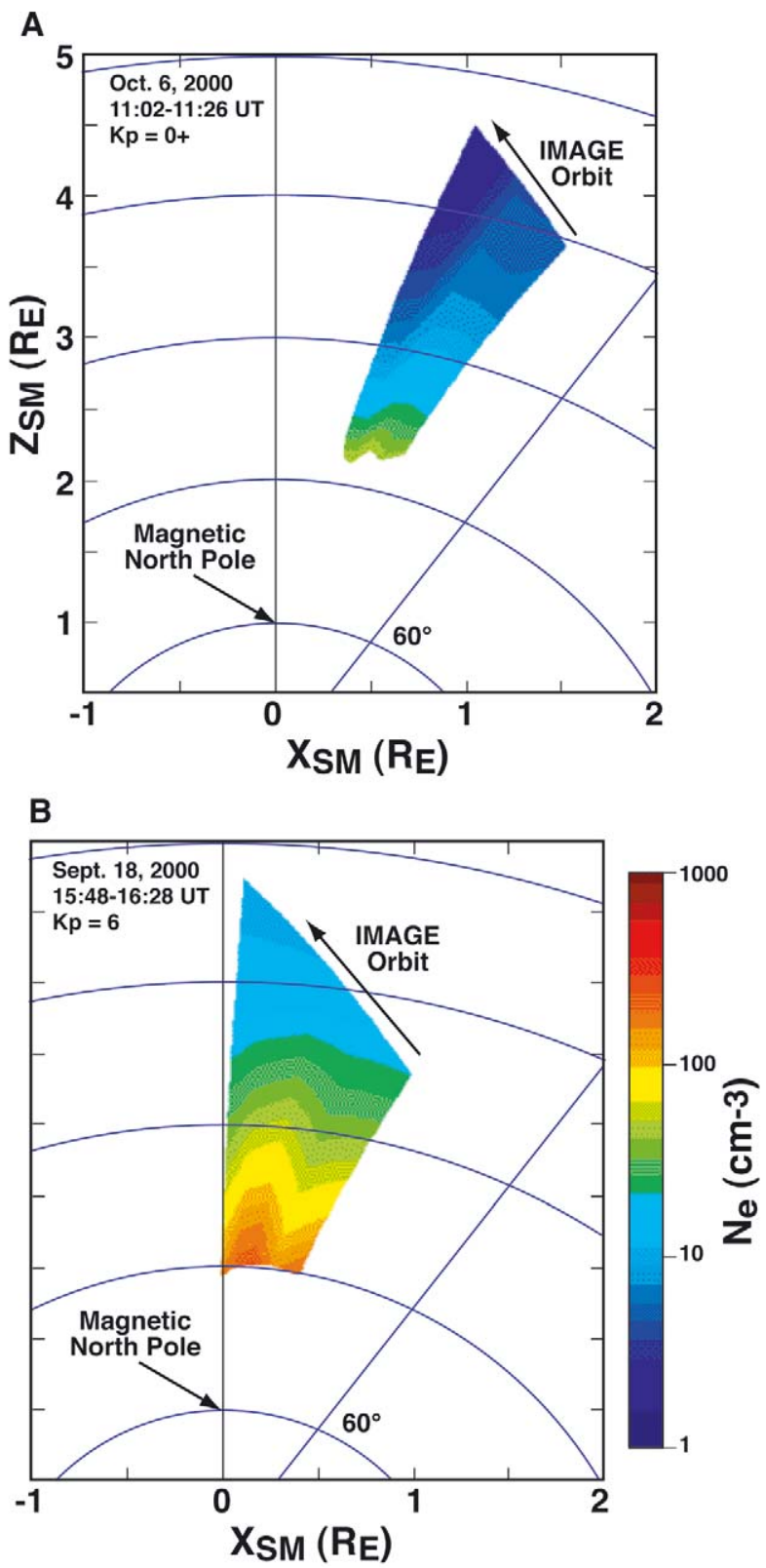

Figure 8. Two RPI passes showing the density structure of the polar cap derived by the inversion of the observed X-mode echoes for $K_{p}=0+\left(\right.$ panel $A$ ) and $K_{p}=6$ (panel B). These results illustrate the drastic changes that can occurs in the polar cap density structure during quiet and disturbed times. 


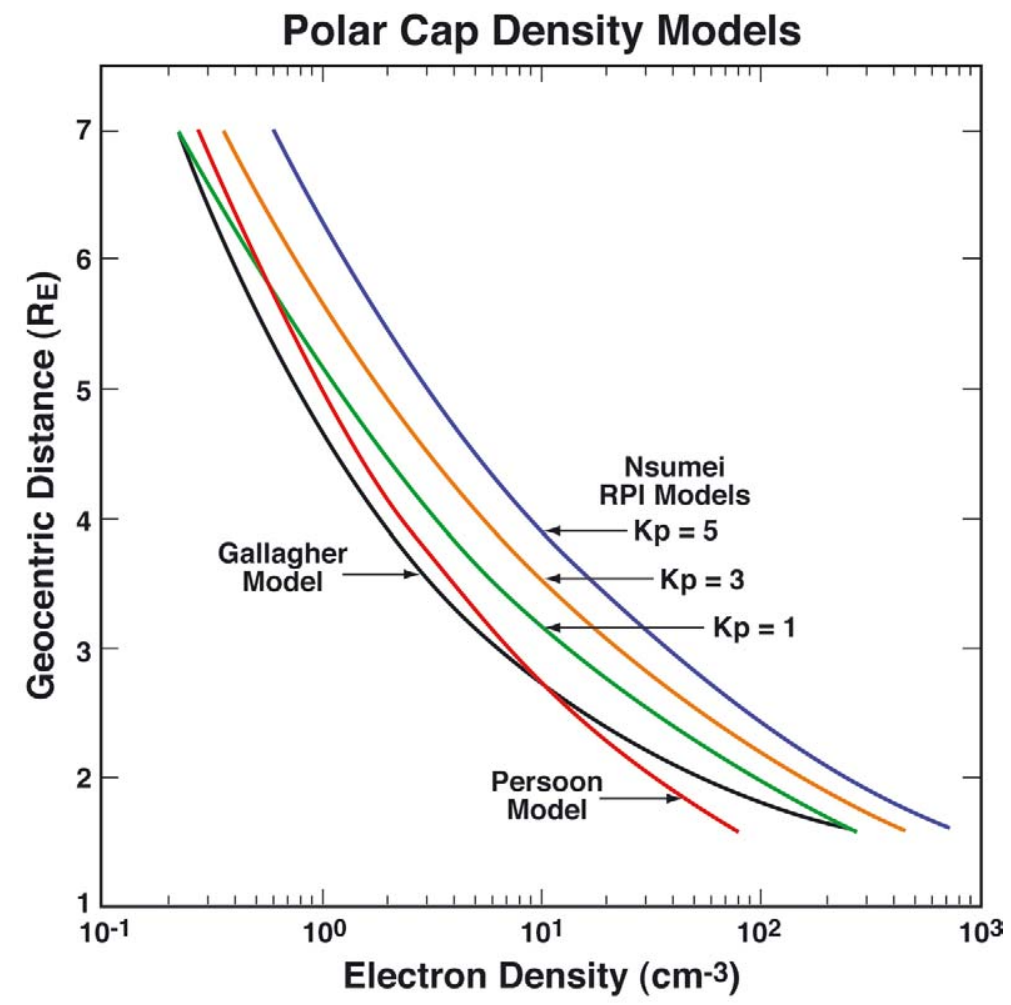

Figure 9. RPI polar cap density models for three separate $\mathrm{K}_{\mathrm{p}}$ ranges derived from 108 plasmagrams. The average polar cap densities for low $\mathrm{K}_{\mathrm{p}}$ compares reasonably well with the previously published model results of Gallagher and Persoon, however, the RPI model densities for large $\mathrm{K}_{\mathrm{p}}$ indices are significantly higher (by a factor of $\sim 10$ ). The RPI model results provide a much more accurate measure of the polar cap density structure since it has none of the drawbacks of the previous results. The RPI data were from a several month period just after solar maximum.

Ion heating at the equator, and therefore enhanced ion pressure, will impede ionospheric flow into the equator giving rise to an increase in the density gradient along at the mid-latitudes.

\subsection{Polar Cap Echoes}

Using RPI X-mode guided echoes over the polar cap and the density inversion technique (described above), the polar cap $\mathrm{N}_{\mathrm{e}}$ distribution can be determined. Nsumei et al. (2002) analyzed 108 plasmagrams with clear polar cap echo traces in the northern hemispheric above $\sim 70^{\circ}$ invariant latitude. As an example of the type of results obtained, Figure 8 panels $\mathrm{A}$ and $\mathrm{B}$, show the two-dimensional polar cap density structures from two separate IMAGE orbit segments over the polar cap when the $K_{p}$ index was $0+$ and 6 , respectively. Each of these orbit segments is created from the inversion of echo traces from 5 consecutive plasmagrams. It is 
clear from these observations that the polar cap $\mathrm{N}_{\mathrm{e}}$ can increase, by nearly a factor of 10, between low (panel A) and high (panel B) $K_{p}$ values. Significant latitudinal $\mathrm{N}_{\mathrm{e}}$ variations were detected on the two passes shown in Figure 8.

Nsumei et al. (2003) created an empirical model as a function of $\mathrm{K}_{\mathrm{p}}$ by appropriately binning and averaging the RPI $\mathrm{N}_{\mathrm{e}}$ data. The result of this analysis is shown in Figure 9 along with a comparison of the Persoon et al. (1983) and Gallagher et al. (2000) polar cap models. The Persoon, Gallagher, and Nsumei $K_{p}=1$ models agree relatively well. However, the RPI polar cap model clearly shows the dependence of the polar cap densities with increasing geomagnetic index $\mathrm{K}_{\mathrm{p}}$. The analysis limitations in the Persoon and Gallagher models primarily involve interpretation of wave propagation cutoffs and particle measurement under low densities and spacecraft charging conditions, all of which underestimates the polar cap $\mathrm{N}_{\mathrm{e}}$. The RPI echo observations have no such limitations and, for the first time, provide a much more realistic determination of the polar cap densities. It is important to note that the RPI measurements were taken near solar maximum while the Persoon and Gallagher models were derived from observations that range over the entire solar cycle. This may account for some of the difference in the models. Because of RPI's ability to obtain the polar cap density structure during individual passes it is certain that these measurements will shed new light on the processes that governs polar wind outflow and contributions of plasmas from auroral or polar cusp sources under different geomagnetic conditions, which was not possible before. These studies are underway.

\subsection{Magnetopause Boundary Layer Echoes}

Prior to the launch of IMAGE much controversy arose concerning whether RPI would be able to observe the magnetopause [see Calvert et al., 1995, 1997; Greenwald, $1997 \mathrm{a}, \mathrm{b}]$. At the time of this writing the RPI team has just begun to analyze echoes from the magnetopause boundary layer [see Fung et al., 2002]. Figure 10 shows three RPI plasmagrams during times when IMAGE was near $7 \mathrm{R}_{\mathrm{E}}$ radial distance, descending from apogee. Each plasmagram clearly shows the plasmaspause ( $\sim 5 \mathrm{R}_{\mathrm{E}}$ distant) and the magnetopause boundary layer echoes ( $\sim 3 \mathrm{R}_{\mathrm{E}}$ distant). The time sequence of plasmagrams in Figure 10 reveals the decreasing distance to the plasmapause and the increasing distance to the magnetopause. From these measurements a number of characteristics of the boundary layer and magnetopause can be determined.

The magnetopause boundary layer echoes always show a considerable amount of range spreading indicating that plasma density irregularities are common in this region as has been reported by Larson and Parks (1992) and others. The diffuse nature of the boundary layer echoes in Figure 10 are due to multiple direct reflections in sharp contract to the more well defined guided echoes as discussed in section 2.1. The rapid drop-off of the trace at high frequencies (as labeled in Figure 10) not only provides us with the distance to the magnetopause but also 


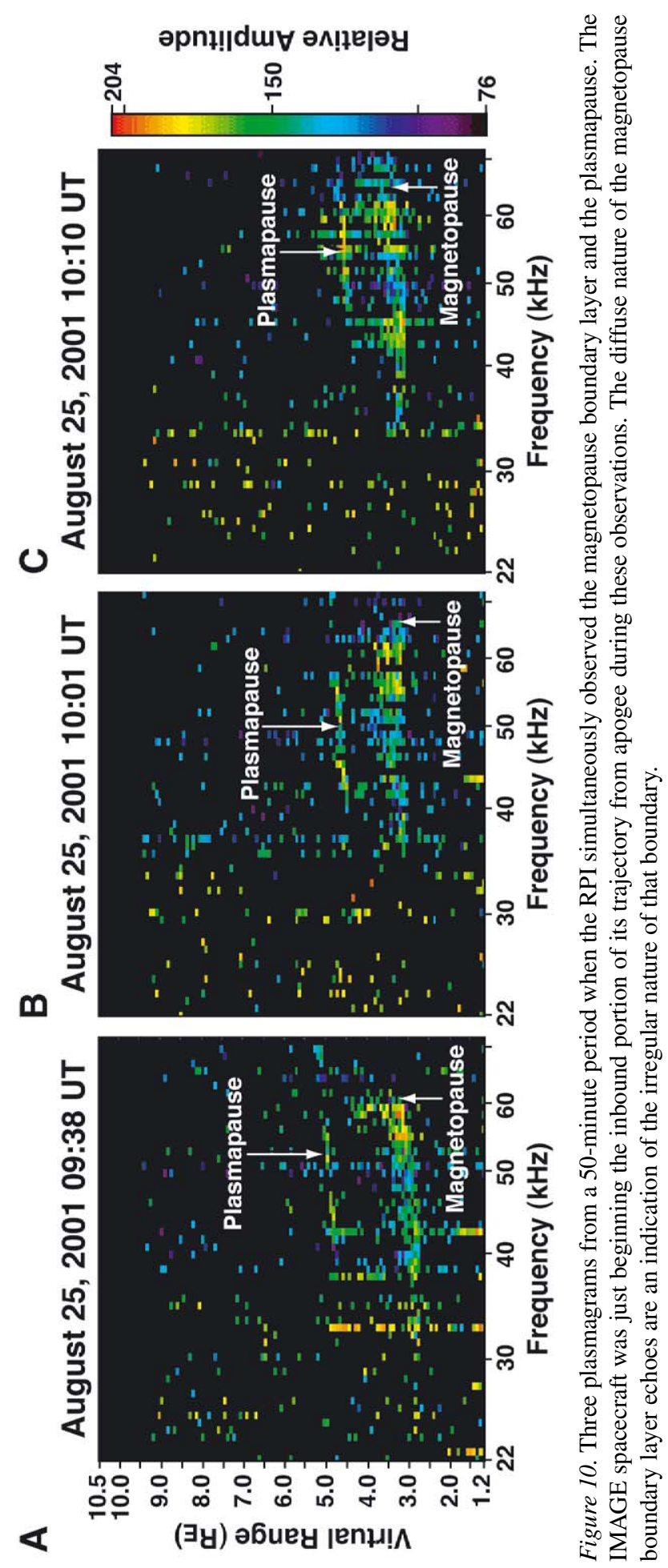


indicates the plasma frequency and therefore its maximum density. The plasma frequency at the magnetopause, assuming O-mode propagation, ranges from 60 to nearly $68 \mathrm{kHz}$ corresponding to a density of $\sim 45-55 \mathrm{~cm}^{-3}$. Over the entire period of 50 min when RPI observed the boundary layer, the nearly horizontal nature of these echoes in the plasmagrams, like those of the plasmapause echoes also shown, indicates a very steep increase in density at the boundary layer starting at about $\sim 18 \mathrm{~cm}^{-3}$ and quickly rising up to the magnetopause density. In addition, the long time period of these observations indicate that the magnetopause boundary layer was relatively stable with its motion confined to within less than about $1 \mathrm{R}_{\mathrm{E}}$. Much more analysis is currently being done on these echoes.

\subsection{Local Plasma Resonance Observations}

The RPI is also a relaxation sounder that generates and detects short-range electrostatic wave echoes [e.g., see Benson, 1977] at the various resonance frequencies or normal modes of a plasma. Like many other missions that have preceded IMAGE (e.g. GEOS 1, ISEE 1) a relaxation-sounder permits accurate determination of the local $\mathrm{N}_{\mathrm{e}}$ and magnitude of the magnetic field (B) at the spacecraft when the resonances have been properly identified. The advantage of the RPI over other magnetospheric relaxation sounders is that it has enough power to generate the long-range electromagnetic echoes (as described in the previous sections) that can uniquely identify the $\mathrm{X}$ and $\mathrm{O}$ mode local cutoff frequencies and thereby allow for the proper identification of all the natural plasma resonances observed.

The local $\mathrm{N}_{\mathrm{e}}$ and $\mathbf{B}$ are determined mainly from the measured frequencies of the sounder-stimulated plasma resonances at $\mathrm{f}_{\mathrm{p}}, \mathrm{f}_{\mathrm{g}}$, and $\mathrm{nf}_{\mathrm{g}}$ (where $\mathrm{n}=2,3$, and larger values). Other resonances, such as the $\mathrm{Q}$ and $\mathrm{D}$ resonances, can also be used to determine these parameters but only when care is taken to properly identify the resonances at frequencies both above the upper hybrid resonance frequency $\left(f_{\text {uhr }}\right.$ ) and below the plasma frequency. Use of the resonance measurements can allow for the determination of the $\mathrm{f}_{\mathrm{p}}$ to within $\sim 1 \%$ and $\mathrm{f}_{\mathrm{g}}$ to within $\sim 0.1 \%$

Figure 11 shows two plasmagrams with plasma resonances taken near apogee one orbit apart just before (panel A) and during (panel B) a geomagnetic storm using the $\mathrm{Z}$ axis antenna. Figure 11 is the result of an extensive resonance analysis process that begins by resorting the RPI data with respect to the gyrofrequency harmonics (see Benson et al., 2003 for more details on this technique). Since all the $\mathrm{Q}$ and $\mathrm{D}$ resonances have a gyrofrequency dependency then they too can be identified in this new coordinate system. D resonances appear below the plasma frequency and $\mathrm{Q}$ resonances appear above the plasmafrequency. The resulting identified resonances are then plotted onto the plasmagram to create Figure 11.

The only resonances measured in panel A of Figure 11 are from the harmonics of $f_{g}$ whose value has been determined to be $2.375 \mathrm{kHz}$. In addition, the local $f_{p}$ must be below $6 \mathrm{kHz}$ since that is the frequency at the start of the sounding 


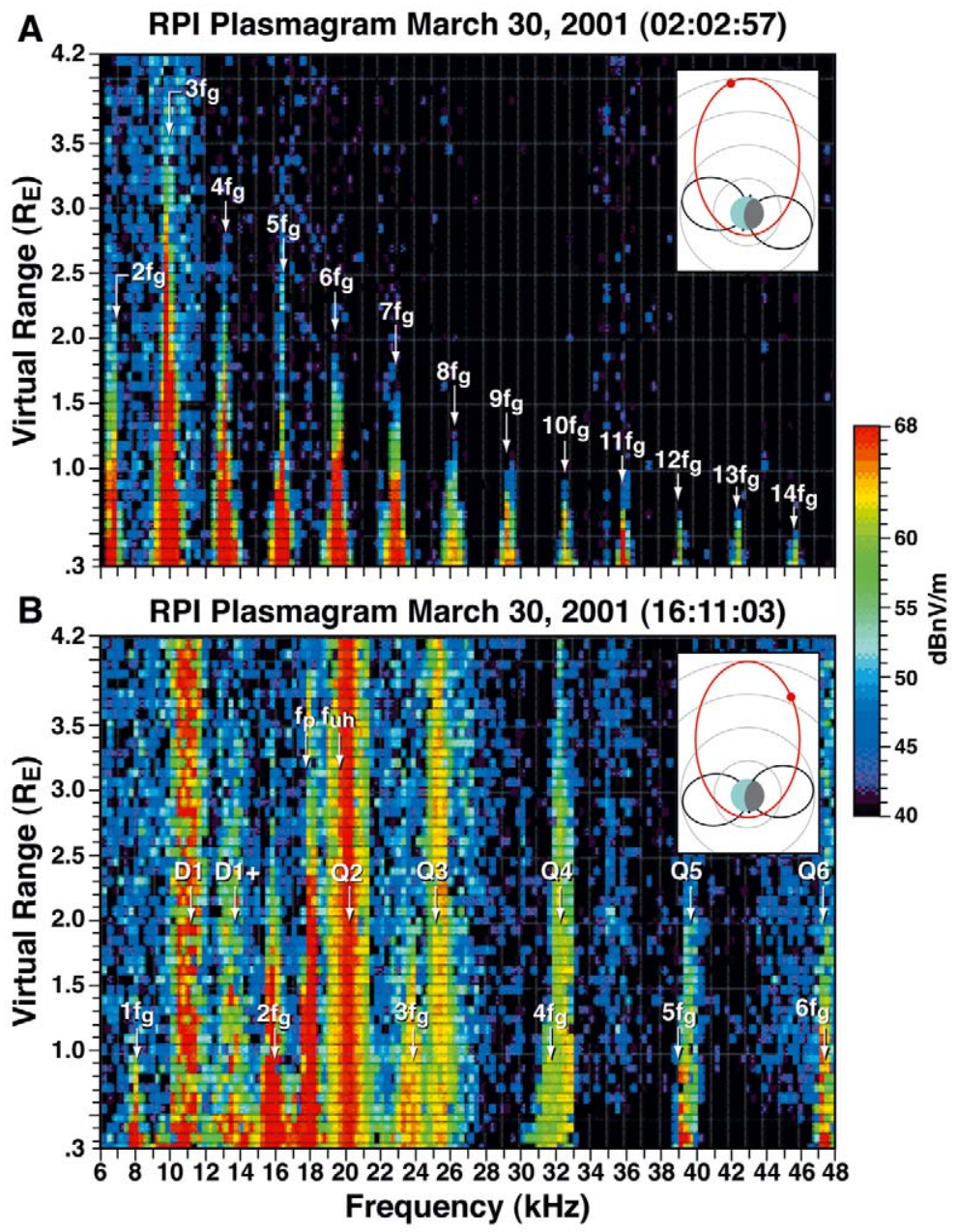

Figure 11. Two RPI plasmagram showing the local plasma resonances conditions near apogee on consecutive orbits prior (panel A) and during (panel B) a large geomagnetic storm. Panel B clearly shows both Q and D resonances. RPI can obtain accurate values of geomagnetic field strength (within a few tenths \%) and $\mathrm{N}_{\mathrm{e}}$ (within a few \%). This figure was adapted from Benson et al. (2002).

sequence. The observed resonances in panel B of Figure 11, one orbit later, are significantly different and show $\mathrm{f}_{\mathrm{p}}, \mathrm{nf}_{\mathrm{g}}, \mathrm{Q}$, and $\mathrm{D}$ resonances. These storm time observations show that $\mathrm{f}_{\mathrm{g}}$ is now over $8 \mathrm{kHz}$ and the observed $\mathrm{f}_{\mathrm{p}}$ is $\sim 17.4 \mathrm{kHz}$. The observed $\mathrm{Q}$ resonances, or Bernstein mode waves, have group velocities nearly matched to the spacecraft, and have been observed in the ionosphere and magnetosphere [see Benson et al., 2003 and references therein] at frequencies between the $\mathrm{f}_{\mathrm{g}}$ harmonics and above $\mathrm{f}_{\text {uhr }}$. Prior to the launch of IMAGE, the D resonances had only been reported in the topside ionosphere at frequencies between the $f_{g}$ harmonics and below $f_{\text {uhr }}$ [see Nelms and Lockwood, 1967]. There has been an on-going 
controversy as to the existence in planetary magnetosphere of the D resonances but it is now clear from the RPI resonance observations that these D resonances are similar to those stimulated by topside sounders in spite of the large differences in the electron temperatures. In light of the intense D resonances easily observed by RPI, previous published results from other magnetospheric relaxation sounders may need to be re-examined for the $\mathrm{D}$ resonances.

\section{Passive Wave Observations}

\subsection{RPI AND EUV COMPARISONS}

In addition to the radio sounding capabilities of RPI, the instrument also regularly makes passive wave measurements. These measurements are needed to provide, not only, context to the radio sounding but can also be compared with observations from other instruments such as the Extreme Ultraviolet (EUV) imager of the plasmasphere [Sandel et al., 2001]. The EUV instrument on IMAGE uses resonance scattering of $\mathrm{He}^{+}$at $30.4 \mathrm{~nm}$ to observe the plasmasphere. $\mathrm{He}^{+}$is typically the second most abundant ion in the plasmasphere. The time resolution of an EUV image is 10 min with typically a spatial resolution of $0.1 \mathrm{R}_{\mathrm{E}}$ at apogee. RPI can be used to measure the $f_{p}$ and therefore, $N_{e}$, which must be equal to the total ion density. Several studies have made a number of these comparisons to determine how the $\mathrm{N}_{\mathrm{e}}$ and $\mathrm{N}_{\mathrm{He}+}$ are related and have found some very interesting results.

Panel A of Figure 12 is a frequency time spectrogram of RPI passive wave measurements. As typically observed in wave spectrograms, the local $\mathrm{f}_{\text {uhr }}$ emission clearly shows the sudden change in density at the plasmapause. The red line in Figure 12 is a model $f_{g}$ (IMAGE does not carry a science magnetometer) and the white line is the $f_{p}$ from the plasmasphere model of Chappell et al. (1970). Visible in many of the RPI spectrograms are regions of enhanced $\mathrm{N}_{\mathrm{e}}$ in the plasma tough region (labeled $B$ in panel A of Figure 12) that appear outside the model $f_{p}$ region. These enhanced densities are similar to the so-called 'detached' plasmaspheric material that has been observed for more than 30 years by a variety of mostly equatorial missions. These enhancements have been found predominately in the dusk sector, often after moderate to disturbed magnetic activity conditions and can have a variety of sized and densities.

With the unique EUV images and the RPI observations these detached plasma regions have been compared by Garcia et al. (2003). A schematic of the results of that study is shown in panel B of Figure 12. EUV images of the plasmasphere are made when the IMAGE spacecraft is at high altitudes $(L>10)$ over the northern polar cap and must be mapped with respect to the IMAGE trajectory at other times. The EUV image in panel B of Figure 12 has been modified to provide a perspective view combined with the trajectory of IMAGE through the plasmasphere and the in situ observations of RPI. The EUV observations have confirmed the existence 


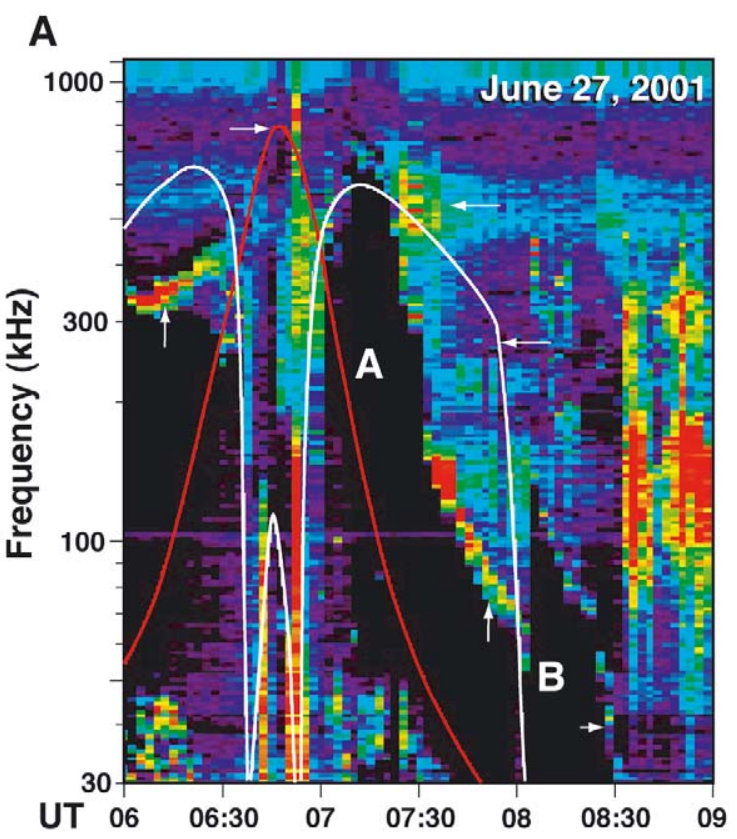

B

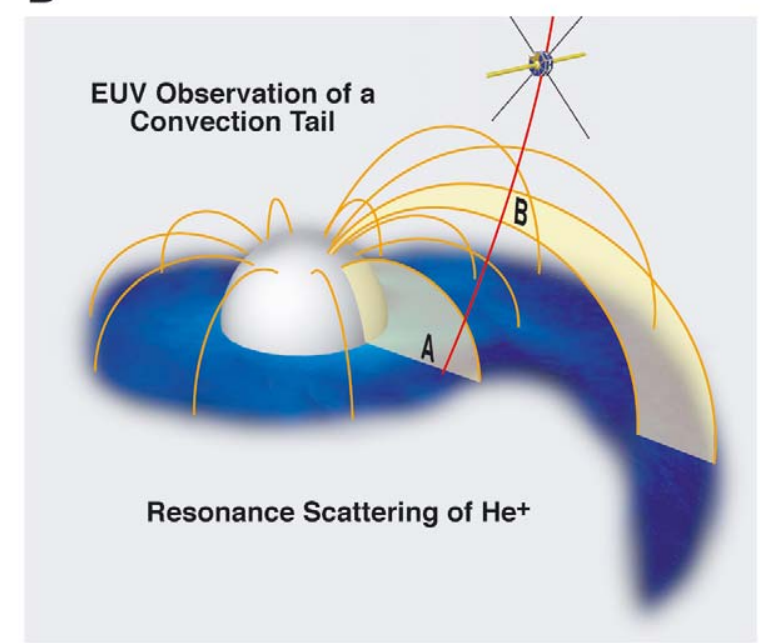

Figure 12. Panel A is a frequency-time spectrogram from RPI during its passage through the plasmasphere on June 27, 2001. The red line is the model $f_{g}$ and the white line is the model $f_{p}$. The high-density plasma in the plasmasphere is highlighted by the observed $\mathrm{f}_{\text {uhr }}$ local electrostatic emissions. Panel B is a schematic of the IMAGE orbit during its passage through the plasmasphere on the dusk side showing the field-lines threading though the outer plasmasphere. Sections labeled A and B refer to corresponding regions in both panels and show that the RPI observed the high latitude extension of a plasmaspheric tail feature as observed by the EUV instrument. This figure was adapted from Garcia et al. (2003). 
of plasmaspheric convection tails [see Sandel et al., this issue] originally proposed by Grebowsky [1970]. The RPI observations of these 'detached' plasma regions were found by Garcia et al. (2003) to be the high latitude extensions of plasma convection tails as shown in panel B of Figure 12. These observations show that the extension of the plasma convection tails occurs along the entire L shell and therefore these tails are not strictly pancake distributions of cold plasma mirroring around the magnetic equator.

Another important study comparing RPI and EUV observations was performed by Goldstein et al. (2002). The comparisons are aimed at comparing the $\mathrm{He}^{+}$edge observed in EUV images with that of the actual plasmapause as measured by RPI. Panel A of Figure 13 is a typical EUV image of the $\mathrm{He}^{+}$plasmasphere on June 24, 2000 at 06:43 UT. The Earth's shadow can easily be seen extending to the upper left corner of the image indicating that local noon points to the lower right hand corner. At most local times a very distinct edge of $\mathrm{He}^{+}$emission can be found and the resulting edge is mapped to the equatorial plane along $\mathrm{L}$ as shown in panel $\mathrm{B}$. The X mark in panel B is the location of the rapid density gradient at the plasmapause as determined by in situ RPI measurements. In this case the agreement is excellent. Goldstein et al. (2002) performed a statistical study of inbound and outbound plasmapause crossings extending over the month of June 2001. The results of that study are shown in panel C of Figure 13, which is a plot of the L shell of the EUV plasmapause with the L shell of the RPI observed plasmapause. The correlation coefficient was found to be 0.86 . This high degree of correlation helps justify the assumption that the $\mathrm{He}^{+}$edge coincides with the plasmapause. Other examples of plasmapause crossings were found in which no sharp boundary was observed in EUV data. The corresponding RPI measurements showed a very gradual plasmapause. Goldstein et al., (2002) also determined that the lower $\mathrm{N}_{\mathrm{e}}$ threshold for EUV was about $40 \mathrm{~cm}^{-3}$.

\subsection{Kilometric Continuum Source Region}

RPI has made significant progress in finding the source region and emission cone characteristics of the kilometric continuum radiation. Continuum radiation is observed over a very broad frequency range from as low as $5 \mathrm{kHz}$ [Gurnett, 1975] to as high as $800 \mathrm{kHz}$ [Hashimoto et al., 1999]. The highest frequency component of the continuum radiation from 100 to $800 \mathrm{kHz}$ has been referred to as 'kilometric' continuum [Hashimoto et al., 1999]. Hashimoto et al. [1999] also found that kilometric continuum was primarily observed when Geotail was at a magnetic latitude less than about $15^{\circ}$. Prior to IMAGE, kilometric continuum was believed to be generated deep inside the plasmasphere and the conditions for which it was generated were largely unknown [Hashimoto et al., 1999].

Panel A of Figure 14 is a frequency versus time spectrogram from RPI passive measurements on April 8, 2001 during a time when IMAGE was moving nearly in the noon-midnight meridian through the dayside and nightside plasmasphere (see 

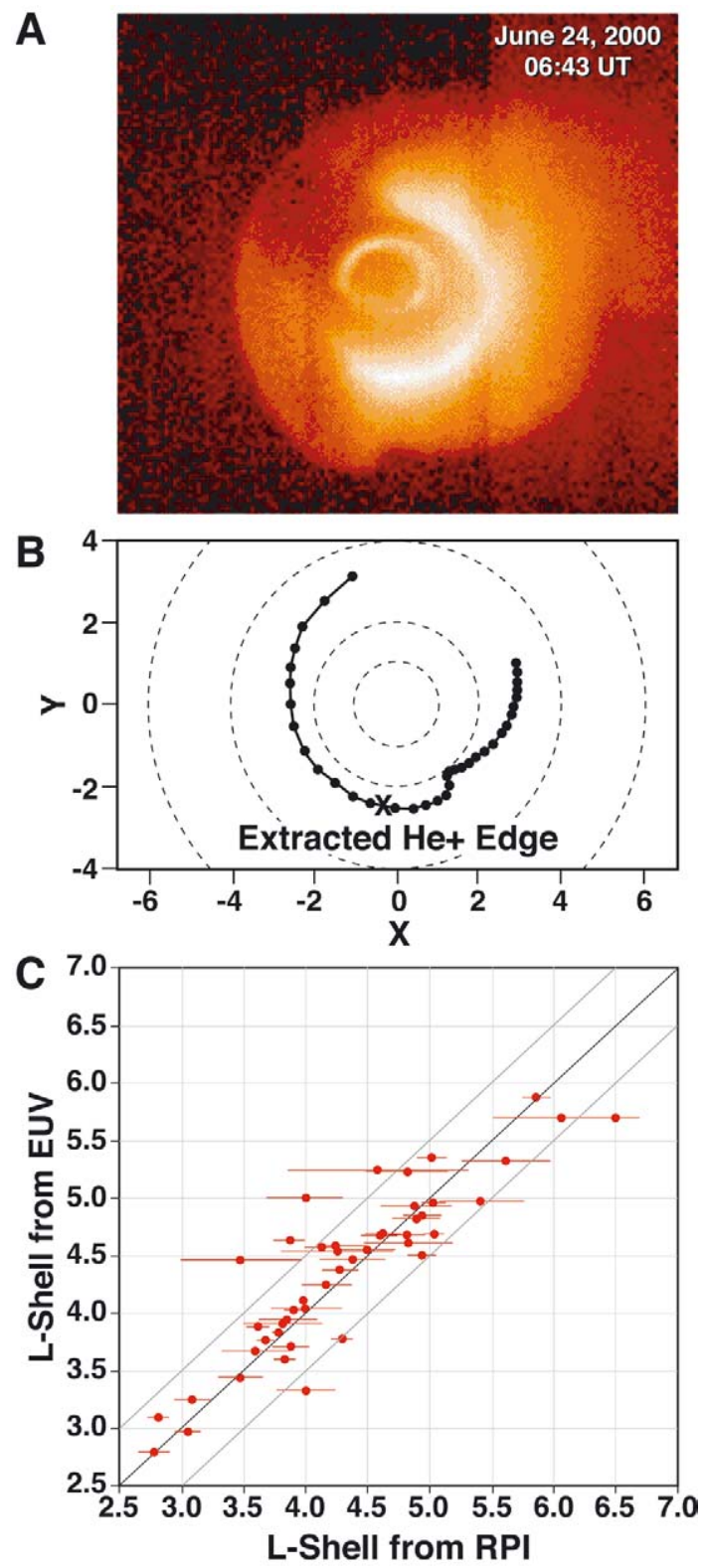

Figure 13. Panel A is an EUV observation of $\mathrm{He}^{+}$resonance scattering of the plasmasphere on June 24, 2000 at 06:43 UT. Panel B is the sharp boundary observed from EUV and used in comparison with the RPI plasmapause crossing results. The cross indicates the $\mathrm{L}$ shell of the plasmaspause as observed by RPI during it passage through the plasmasphere. Panel $\mathrm{C}$ is a plot of the $\mathrm{L}$ value of the plasmapause, as measured by RPI versus those measured by EUV from over 40 events. This figure was adapted from Goldstein et al., (2002). 

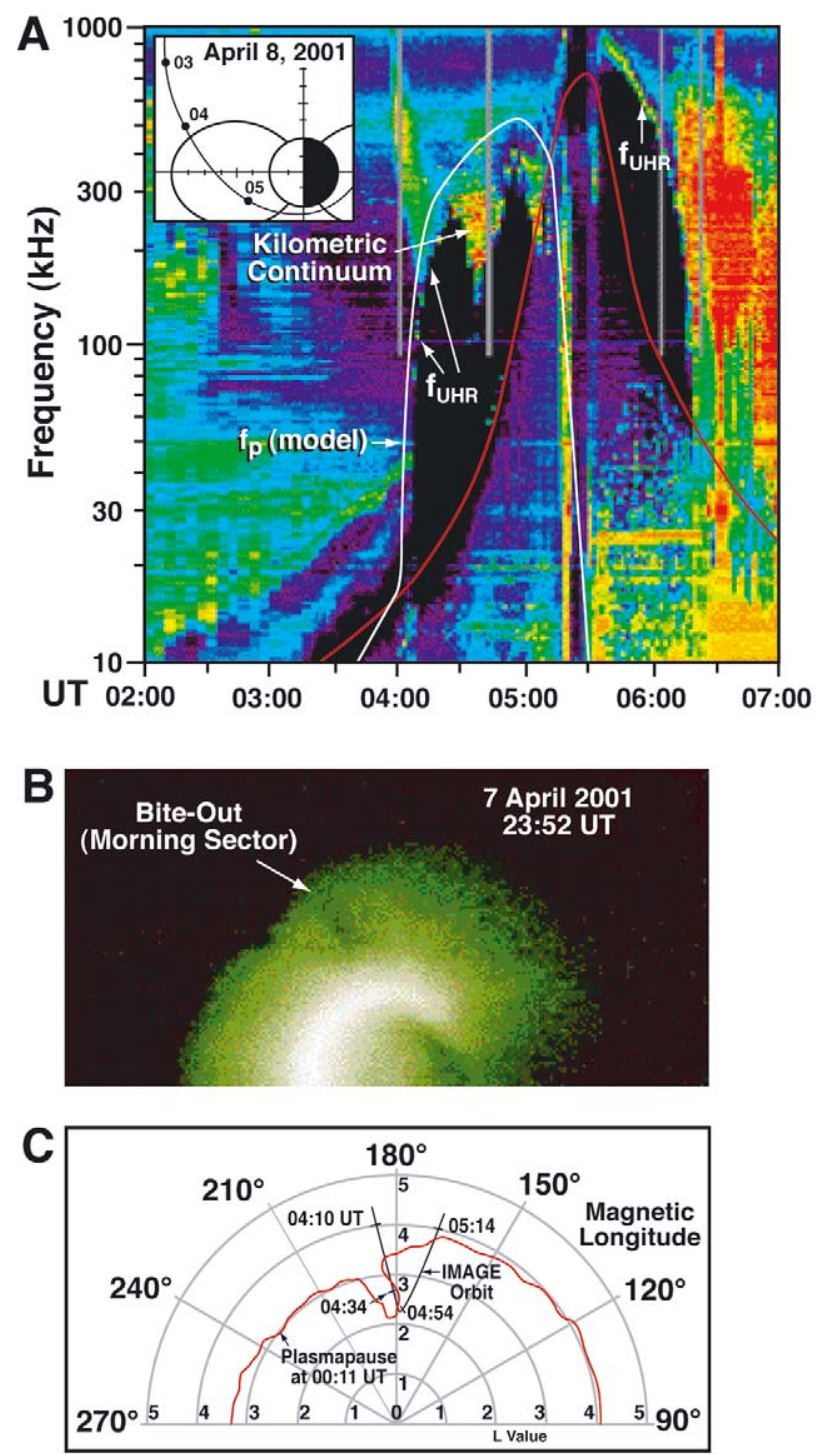

Figure 14. Panel A is a RPI spectrogram during the passage of the IMAGE spacecraft through the plasmasphere nearly in the noon-midnight meridian plane on April 8, 2001. The deviation of the $f_{u h r}$ emission from the model $f_{g}$ from 04:25 to 04:50 UT indicates that IMAGE passed through a deep density depression in the dayside plasmasphere. Panel B is an EUV He${ }^{+}$image of the plasmasphere showing a distinct bite-out region taken several hours before the passage of IMAGE through the plasmasphere. Assuming the bite-out region continues to corotate, panel $\mathrm{C}$ shows that the deep density depression observed by RPI was the bite-out structure previously observed by EUV. As shown in panel A, kilometric continuum was observed to be confined to the bite-out region. This figure was adapted from Green et al., (2002). 
insert). The RPI instrument observes kilometric continuum radiation in a plasmaspheric density depression on the dayside as delineated by the difference between the model $f_{p}$ and the observed $f_{u h r}$. Observations of kilometric continuum in density depletions, like those shown in panel A, have also been observed by a number of other missions with wave instruments [e.g. Carpenter et al., 2000].

When compared with measurements by the EUV instrument on IMAGE these passive wave measurements by RPI have provided a new insight into the source region of kilometric continuum [Green et al., 2002; Green et al., 2003]. Panel B of Figure 14 is an image of the plasmasphere taken at 23:52 UT on April 7, 2001 by EUV. The direction to the sun is in the upper left in this image. The distinct biteout feature (also called a plasmaspheric notch), shown in panel B, was observed for approximately 5 hours in repetitive EUV images and found to corotate with the plasmasphere. The EUV instrument is routinely turned off during perigee passages of IMAGE below about an L of 8 so was not imaging during the time of the RPI observations in panel A, however, the mapping of the IMAGE trajectory with respect to the corotating bite-out structure is shown in Figure 14 panel C. From these results, Green et al. (2002) proposed that kilometric continuum would be generated at the newly established plasmapause deep within plasmaspheric bite-out structures. At this time it is not known how these newly discovered plasmaspheric bite-out structures are created.

In a follow-up study by Green et al. (2003), IMAGE and Geotail observations of kilometric continuum and EUV observations of the plasmasphere were combined to confirm these initial results. Figure 15 shows a Geotail spectrogram of the narrow band kilometric continuum emissions (panel A) and its position relative to a simultaneously observed EUV bite-out structure (panel B). Geotail's position during these observations was within $10^{\circ}$ of the magnetic equator at nearly $20 \mathrm{R}_{\mathrm{E}}$. The plasmapause determined from EUV data including the bite-out structure is shown in panel B at the same magnetic longitude as the Geotail observations of the bite-out. Ray tracing calculations by Green et al. (2002), shown in the inset of panel $\mathrm{B}$, illustrates that the kilometric continuum source region lying deep within the plasmaspheric bite-out provides a significant propagation effect to the ray paths that will confine the kilometric continuum emission cone in longitude to approximately fill the bite-out structure.

A summary of the results from the RPI observations of kilometric radiation is shown in Figure 16 with the emission cone of auroral kilometric radiation. Since kilometric continuum is observed in the same frequency range as AKR, earlier surveys using instruments with poor frequency resolution could have easily mistaken kilometric continuum for AKR [see for example Figure 5 in Green et al., 1977]. Unlike AKR, which is generated in the nightside auroral zone and beamed primarily tailward, kilometric continuum emissions would be emitted into relatively flat emission cones in the magnetic equator that co-rotate with the plasmasphere over all local times or until the emission ceases. 

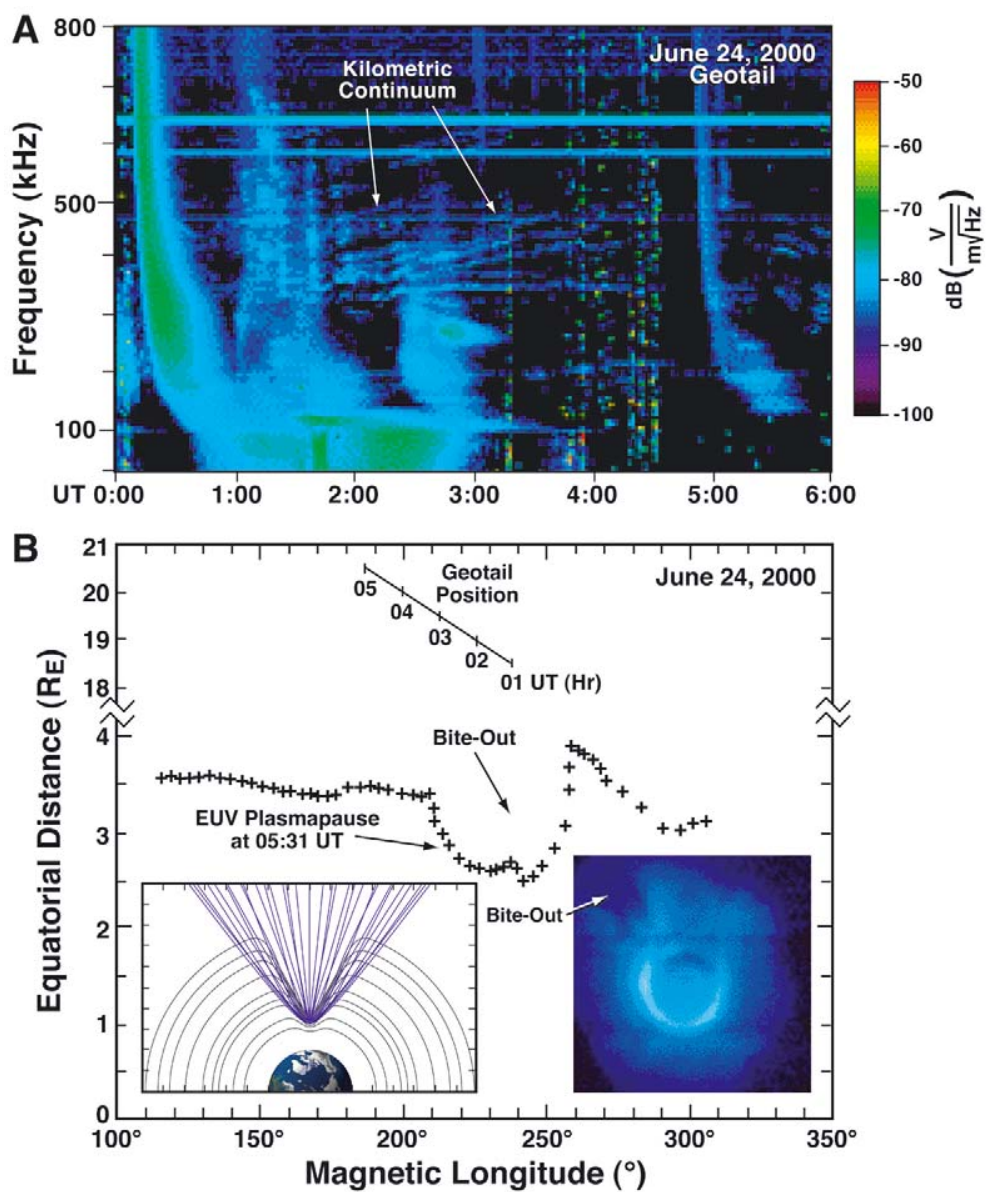

Figure 15. Panel A is a frequency time spectrogram from the Geotail spacecraft showing the banded structure of the kilometric continuum stretching from about 01:00 to 05:00 UT over the frequency range above $100 \mathrm{kHz}$ to $600 \mathrm{kHz}$. Panel B shows the simultaneous EUV observations (inset) from IMAGE of a large bite-out structure plotted in magnetic longitude and compared to the position of Geotail during its observations of kilometric continuum of panel A. Ray tracing results (inset in panel B) show that a source of kilometric continuum at the $f_{u h r}$ frequency deep inside the bite-out structure produces an emission cone largely confined to the bite-out structure which is also consistent with the Geotail observations. This figure was adapted from Green et al. (2003).

\subsection{RPI TRANSMISSIONS AND RECEPTION FROM WIND AND CLUSTER}

High frequency transmissions of RPI in the sounding mode, when received by the plasma wave instruments on remote spacecraft such as Wind or Cluster, are now providing a new opportunity to test the feasibility of radio tomography as a possible imaging technique for use on upcoming constellation missions [e.g. Ergun et al., 2000]. Cummer et al. (2001) have published the results from a series of single 


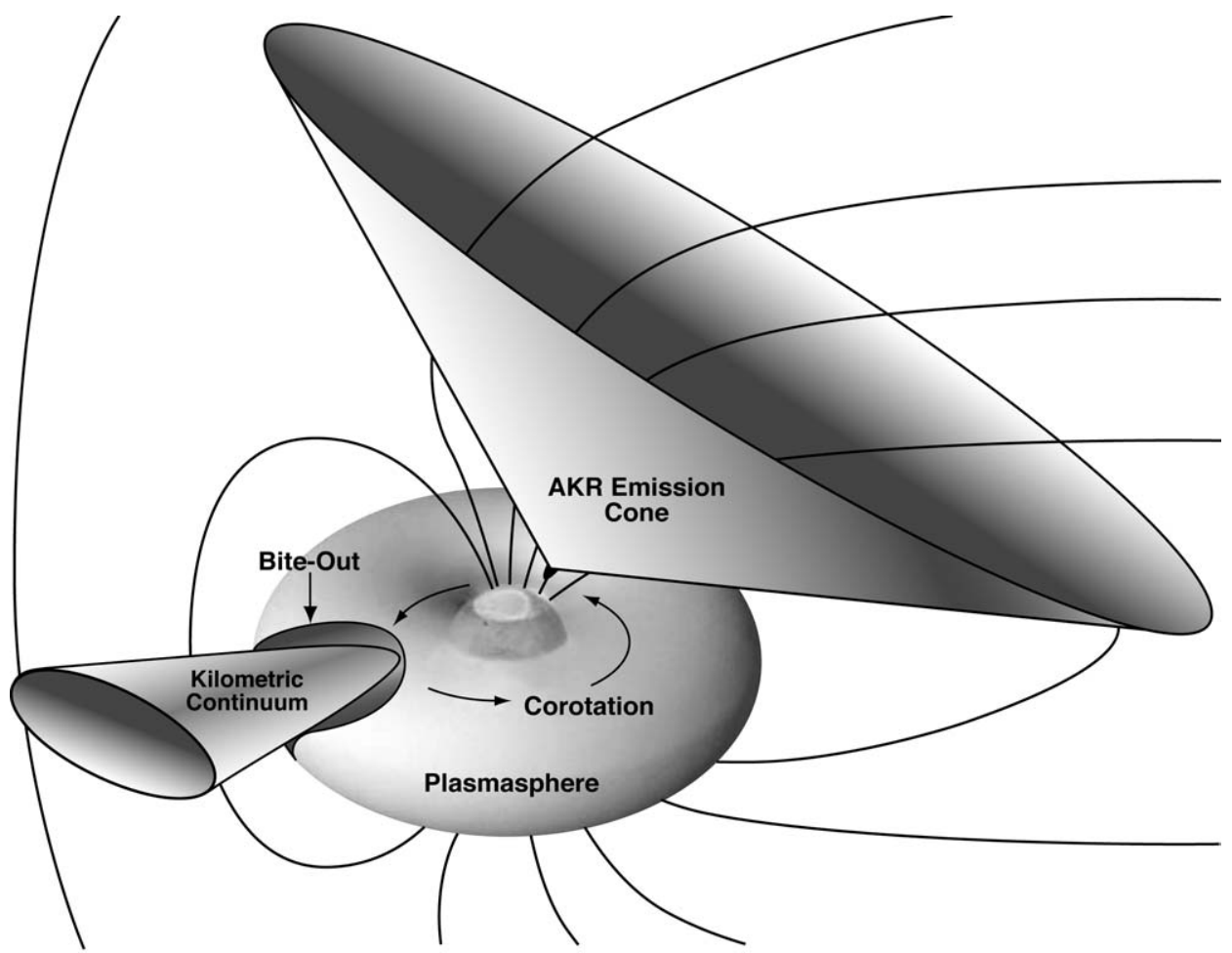

Figure 16. Schematic of auroral and continuum radiation emission cones (Green et al., 2002). The AKR emission cone is tilted back and beamed tailward while the kilometric continuum emission cone has been found to be generated at the plasmapause in bite-out regions of the plasmasphere and beamed into an emission cone typically less than $15^{\circ}$ in latitude around the magnetic equator.

frequency emissions by RPI on IMAGE, with receptions by the Waves instrument on Wind over a distance of about $6 \mathrm{R}_{\mathrm{E}}$.

For the first series of tests, RPI transmissions were at $828 \mathrm{kHz}$ with a repetitive 2-minute cycle of 64 seconds of 0.5 seconds on then 0.5 seconds off followed by 56 seconds of no transmissions. Since RPI was transmitting on only one of its antennas the result, as observed by the Z-axis on the Wind/Waves instrument, should vary sinusoidally. Panel A of Figure 17 shows the Wind/Waves received RPI transmissions from the $\mathrm{Z}$ antenna along with the predicted IMAGE spin pattern based on its $122.7 \mathrm{~s}$ spin period. Within this segment of data the modulation pattern alternated between not matching and matching the predicted spin period. The discrepancy varies with time and is due to the time-varying Faraday rotation of the electric field as it passes through the intervening plasma between the spacecraft. Panel B of Figure 17 shows the change in the path integrated $N_{e}$ and $\mathbf{B}$ measured from the observed Faraday rotation and demonstrates the feasibility of this technique. To obtain images of $\mathrm{N}_{\mathrm{e}}$ over large regions of space additional spacecraft will be needed 

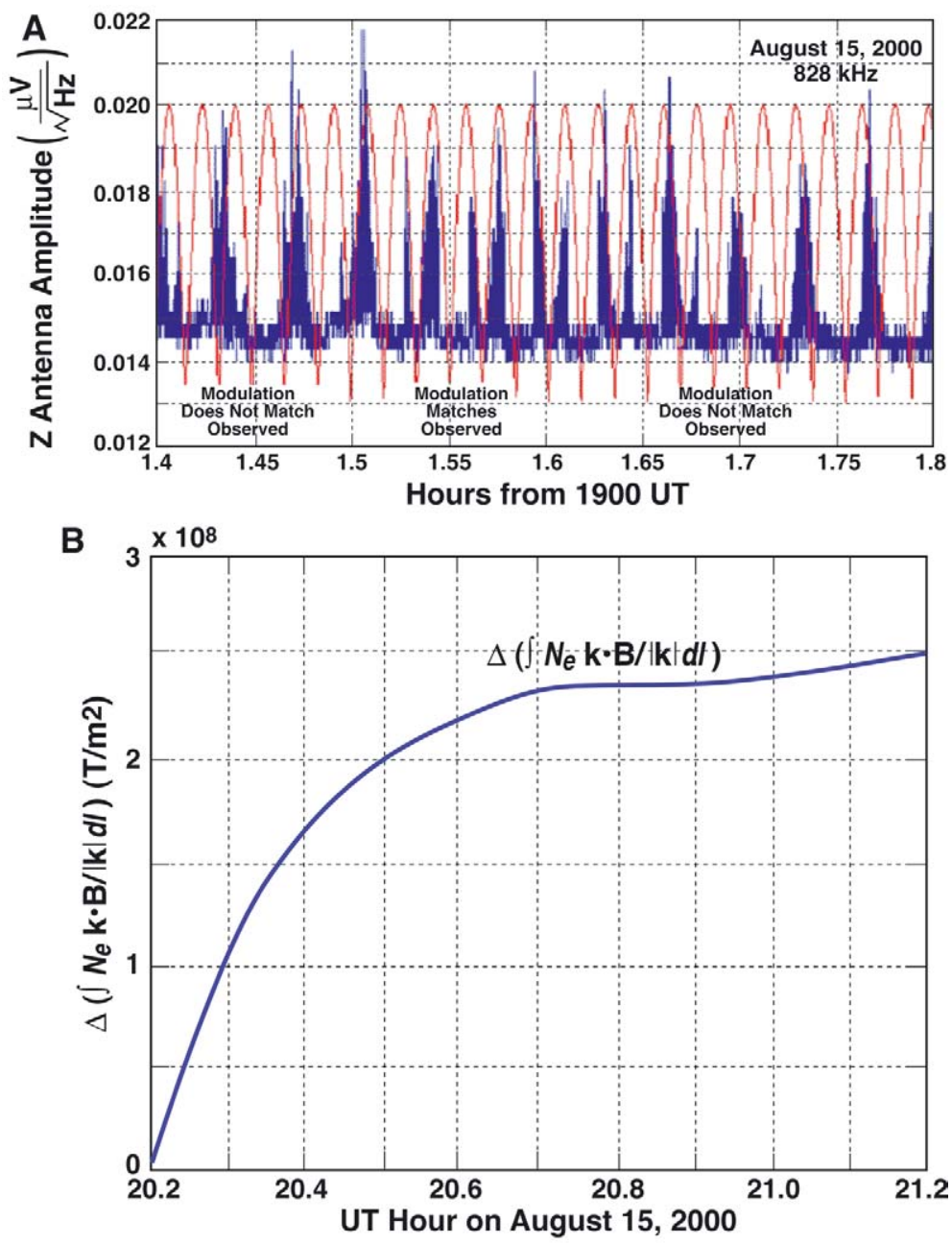

Figure 17. Panel A shows the Wind/Waves instrument amplitude measurements from the $\mathrm{Z}$ antenna of the IMAGE/RPI transmissions over a short period of time. The modeled signal, shown in red, is based on the actual RPI spin period of $122.7 \mathrm{~s}$. The discrepancy between the nulls in the measurements and that from the model is due to the time-varying Faraday rotation of the electric field of the received signal. Panel $\mathrm{B}$ shows the change in the path-integrated $\mathrm{N}_{\mathrm{e}}$ and magnetic field product measured from the observed Faraday rotation over a period of an hour.

making these types of measurements over propagation paths that cross allowing the application of tomographic inversion to yield the final result.

More recently, Cummer et al., 2003 have successful conducted multi-frequency tests with IMAGE/RPI transmitting and Wind/Waves and all four Cluster/Wideband instruments receiving. Each of these experiments clearly show the Faraday rotation of the electric field at each of the frequencies used. 


\section{Conclusions}

The RPI continues to provide a wealth of observations about the electron density structures and boundary locations of many features in the Earth's magnetosphere. RPI sounding observations of echoes can determine the plasmasphere densities at distances greater than $3 \mathrm{R}_{\mathrm{E}}$. These are the first radio soundings at such large radial distances within the magnetosphere. During soundings of the plasmasphere, direct RPI echoes exhibit various amounts of spreading, from $\sim 0.5 \mathrm{R}_{\mathrm{E}}$ to $\sim 2 \mathrm{R}_{\mathrm{E}}$ in virtual range. These observations suggest that the plasmasphere is regularly permeated by field-aligned irregularities with scale sizes ranging from $\sim 200 \mathrm{~m}$ to over $10 \mathrm{~km}$ and plasma density within of $\sim 10 \%$ of background.

One of the surprising results from the RPI is that a large number of guided echoes are observed in the plasmapause region, plasmaspheric notch or bite-outs, in the plasma trough, and over the polar cap. A profile inversion technique for RPI echo traces has been developed that provides a method for determining the electron density $\left(\mathrm{N}_{\mathrm{e}}\right)$ distribution of the plasma from either direct or field-aligned echoes. This technique has enabled the determination of the evolving density structures of the polar cap and the plasmasphere under a variety of geomagnetic conditions.

In addition to the RPI long range echoes, plasma resonance observations from RPI at large radial distances over the polar cap have been made allowing for the determination of the plasma density to within an accuracy of a few percent and the magnetic field intensity within an accuracy of a few tenths of a percent. The plasma resonances have been used to provide the starting points for the inversion process leading to $\mathrm{N}_{\mathrm{e}}$ polar-cap profiles measured by RPI.

Passive measurements from RPI, when combined with observations from the EUV imager, have found kilometric continuum source regions deep within biteout structures in the plasmasphere. In addition, the plasma convection tails are observed to be connected back to the ionosphere along the same L shells with significant amounts of cold plasmaspheric plasma.

RPI has also been used to successfully test the feasibility of magnetospheric tomography during several perigee passages of the Wind spacecraft. Many future multi-spacecraft missions propose to use Faraday rotation to obtain global density pictures of the magnetosphere. The RPI radio transmissions at one and two frequencies have been received by the WIND Waves instrument. Faraday rotation was measured and occurs when the received electric field was observed to rotate with time due to the changing column density of plasma along the line of sight between the spacecraft.

\section{Acknowledgements}

The work at the University of Massachusetts Lowell was supported by NASA under subcontract 83822 with the Southwest Research Institute. The authors would 
also like to gratefully acknowledge the software development work of Ivan Galkin, Grigori Khmyrov, and Alexander Kozlov of UML, and Scott Boardsen of GSFC. Helpful discussions with W. W. L. Taylor and Robert Benson are also acknowledged.

\section{References}

Benson, R.F.: 1997, 'Evidence for the stimulation of field-aligned electron density irregularities on a short time scale by ionospheric topside sounders', J. Atm. and Solar-Terr. Phys. 59, 2281-2293.

Benson, R.F., Osherovich, V.A., Fainberg, J., and Reinisch, B.W.: 2003, 'Classification of IMAGE/RPI-stimulated resonances for the accurate determination of magnetospheric electrondensity and magnetic field values' 108(A5), 1207, doi: 1029/2002JA009589, J. Geophys. Res.

Calvert, W., Benson, R.F., Carpenter, D.L., Fung, S.F., Gallagher, D.L., Green, J.L., Haines, D.M., Reiff, P.H., Reinisch, B.W., Smith, M.F. and Taylor, W.W.L.: 1995, 'The feasibility of radio sounding in the magnetosphere', Radio Science 30, 5, 1577-1615.

Calvert, W., Benson, R.F., Carpenter, D.L., Fung, S.F., Gallagher, D., Green, J.L., Haines, D.M., Reiff, P.H., Reinisch, B.W., Smith, M. and Taylor, W.W.L.: 1997, 'Reply to R.A. Greenwald concerning the feasibility of radio sounding of the magnetosphere', Radio Sci. 32, 1, 281-284.

Carpenter, D.L., Anderson, R.R., Calvert, W. and Moldwin, M.B.: 2000, 'CRRES observations of density cavities inside the plasmasphere', J. Geophys. Res. 105, 23323.

Carpenter, D.L., Spasojevic, M., Bell, T.F., Inan, U.S., Reinisch, B.W., Galkin, I.A., Benson, R.F., Green, J.L., Fung, S.F., Boardsen, S.A.: 2002, 'Small-scale field-aligned plasmaspheric density structures inferred from RPI on IMAGE', 107(A9), 1258, 10.1029/2001JA009199, J. Geophys. Res.

Chappell, C.R., Harris, K.K. and Sharp, G.W.: 1970, 'A study of the influence of magnetic activity on the location of the plasmapause as measured by OGO-5', J. Geophys. Res. 75, 50-56.

Cummer, S.A., Reiner, M.J., Reinisch, B.W., Kaiser, M.L., Green, J.L., Benson, R.F., Manning, R., Goetz, K.: 2001, 'A test of magnetospheric radio tomographic imaging with IMAGE and WIND', Geophys. Res. Letts. 28, 1131-1134.

Cummer, S.A., Green, J.L., Reinisch, B.W., Fung, S.F., Kaiser, M.L., Mutel, R., Pickett, J., Chistopher, I., Gurnett, D.A.: 2003, 'Advances in Magnetospheric Radio Wave Analysis and Tomography', Adv. Space Res. Accepted.

Ergun, R.E. et al.: 2000, 'Feasibility of a multi-satellite investigation of the Earth's magnetosphere with radio tomography', J. Geophys. Res. 105, 361-373.

Fung, S.F., Benson, R.F., Green, J.L., Reinisch, B.W., Haines, D.M., Galkin, I.A., Bougeret, J.-L., Manning, R., Reiff, P.H., Gallagher, D.L., Carpenter, D.L. and Taylor, W.W.L.: 2002, 'Observations of Magnetospheric Plasmas by the Radio Plasma Imager (RPI) on the IMAGE Mission', Adv. Space Res. 30 (10), 2259-2266.

Fung, S.F., Benson, R.F., Carpenter, D.L., Green, J.L., Jayanti, V., Galkan, I.A. and Reinisch, B.W.: 2003, 'Guided Echoes in the Magnetosphere: Observations by Radio Plasma Imager on IMAGE' 30(11), 1589, doi: 10.1029/2002GL016531, Geophys. Res. Letts.

Gallagher, D.L., Craven, P.D. and Comfort, R.H.: 2000, 'Global core plasma model', J. Geophys. Res. 105, 18819.

Garcia, L.N., Fung, S.F., Green, J.L., Boardsen, S., Sandel, B.R. and Reinisch, B.W.: 2002, 'Comparison of IMAGE RPI and EUV observations of plasma density structures outside of the plasmasphere', Accepted J. Geophys. Res. 
Goldstein, J., Spasojevic, M., Reiff, P.H., Sandel, B.R., Forrester, T., Gallagher, D.L. and Reinisch, B.W.: 2002, 'Identifying the plasmapause in IMAGE EUV data using IMAGE RPI in situ steep gradients', Accepted in J. Geophys. Res.

Grebowsky, J.M.: 1970, 'Model study of plasmapause motion', J. Geophys. Res. 75, 4329-4333.

Green, J.L., Gurnett, D.A. and Shawhan, S.D.: 1977, 'The angular distribution of auroral kilometric radiation', J. Geophys. Res. 82, 1825.

Green, J.L., Sandel, B.R., Fung, S.F., Gallagher, D.L. and Reinisch, B.W.: 2002, 'On the Origin of Kilometric Continuum', J. Geophys. Res. 107(A7), 10.1029/2001JA000193.

Green, J.L., Boardsen, S.A., Fung, S.F., Matsumoto, H., Hashimoto, K., Anderson, R.R., Sandel, B.R. and Reinisch, B.W.: 2003, 'Association of Kilometric Continuum Radiation with Plasmaspheric Structures', Submitted to J. Geophys. Res. .

Greenwald, R.A.: January-February 1997a, 'Comment on The feasibility of radio sounding of the magnetopause by W. Calvert et al.', Radio Science 32, 277-280.

Greenwald, R.A.: May-June 1997b, 'Rebuttal to reply by W. Calvert et al.', Radio Science 32, 877879.

Gurnett, D.A.: 1975, 'The Earth as a radio source: The nonthermal continuum', J. Geophys. Res. 80, 2751-2763.

Hashimoto, K., Calvert, W. and Matsumoto, H.: 1999, 'Kilometric continuum detected by Geotail', J. Geophys. Res. 104, 28645-28656.

Huang, X. and Reinisch, B.W.: 1982, 'Automatic calculation of electron density profiles from digital ionograms 2'. True height inversion of topside ionograms with the profile-fitting method, Radio Sci. 17, 837-844.

Larson, N.R., and Parks, G.K.: 1992, 'Motions of particle microstructures in the magnetopause boundary layer', J. Geophys. Res. 97, 10733-10749.

Nelms, G.L., and Lockwood, G.E.K.: 1967, 'Early results from the topside sounder in the Alouette II satellite, Space Research VII', in R.L. Smith-Rose (ed.), North-Holland Publishing Co., Amsterdam, pp. 604-623.

Nsumei, P.A., Huang, X., Reinisch, B.W., Song, P., Vasyliunas, V.M., Green, J.L., Fung, S.F., Benson, R.F. and Gallagher, D.L.: 2003, 'Electron Density distribution over the northern polar region deduced from IMAGE/RPI sounding' 108(A2), 1078, doi: 1029/2002JA009616, J. Geophys. Res.

Park, C.G.: 1974, 'Some features of plasma distribution in the plasmasphere deduced from Antarctic whistlers', J. Geophys. Res. 79, 169-173.

Persoon, A.M., Gurnett, D.A. and Shawhan, S.D.: 1983, 'Polar cap electron densities from DE 1 plasma wave observations', J. Geophys. Res. 88, 10123.

Reinisch, B.W., Haines, D.M., Bibl, K., Cheney, G., Galkin, I.A., Huang, X., Myers, S.H. and Sales, G.S., Benson, R.F., Fung, S.F., Green, J.L., Taylor, W.W.L., Bougeret, J.-L., Manning, R., MeyerVernet, N., Moncuquet, M., Carpenter, D.L., Gallagher, D.L. and Reiff, P.: February, 2000, 'The Radio Plasma Imager investigation on the IMAGE spacecraft', Space Science Reviews, IMAGE special issue, 91, 319-359.

Reinisch, B.W., Huang, X., Haines, D.M., Galkin, I.A., Green, J.L., Benson, R.F., Fung, S.F., Taylor, W.W.L., Reiff, P.H., Gallagher, D.L., Bougeret, J.-L., Manning, R. and Carpenter, D.L.: 2001a, 'First Results from the Radio Plasma Imager on IMAGE', Geophys. Res. Letts. 28, 1167-1170.

Reinisch, B.W., Huang, X., Song, P., Sales, G.S., Fung, S.F., Green, J.L., Gallagher, D.L. and Vasyliunas, V.M.: 2001b, 'Plasma density distribution along the magnetospheric field: RPI observations from IMAGE', Geophys. Res. Letts. 28, 4521-4524.

Reinisch, B.W., Huang, X., Song, P., Sales, G.S., Galkin, S.I., Benson, R., Fung, S.F. and Green, J.L.: 2002, 'Radio Plasma Imager observation of magnetostorm effects on the plasmaspheric density distribution, abstract, $27^{\text {th }}$ General Assembly of the International Union of Radio Science (URSI)', Maastricht, The Netherlands, August 17-24, 2002. 
Sandel, B.R., King, R.A., King, W.A., Forrester, W.T., Gallagher, D.L., Broadfoot, A.L. and Curtis, C.C.: 2001, 'Initial results from the IMAGE Extreme Ultraviolet Imager', Geophys. Res. Letts. 28, 1439-1442.

Sandel, B.R., Goldstein, J., Gallagher, D.L. and Carpenter, D.L.: 2003, 'EUV Observations of the Structure and Dynamics of the Plasmasphere', this issue.

Stix, T.H.: 1962, 'The Theory of Plasma Waves', McGraw-Hill, New York.

Tu, J., Horwitz, J.L., Song, P., Huang, X-Q, Reinisch, B.W. and P.G. Richards, P.G.: 2003, 'Simulating plasmaspheric field-aligned density profiles measured with IMAGE/RPI: Effects of plasmasphere refilling and ion heating', J. Geophys. Res. in press. 